\title{
EXISTENCE RESULTS FOR A POLYMER MELT WITH AN EVOLVING NATURAL CONFIGURATION
}

\author{
J. K. DJOKO \\ Department of Mathematics and Applied Mathematics, \\ University of Pretoria, \\ Pretoria 0002, South Africa \\ jules.djokokamdem@up.ac.za \\ B. D. REDDY \\ Department of Mathematics and Applied Mathematics, \\ and Center for Research in Computational and Applied Mechanics, \\ University of Cape Town, \\ 7701 Rondebosch, South Africa \\ daya.reddy@uct.ac.za
}

\begin{abstract}
We consider a set of equations governing the behavior of a polymer melt which is modeled as a viscoelastic fluid possessing a natural, or stress-free state. The natural configuration is characterized through a symmetric, proper orthogonal intermediate deformation tensor, analogous to the left Cauchy-Green deformation tensor in continuum mechanics. This tensor is required to satisfy an evolution equation. It is shown that the constraint that the intermediate tensor be proper orthogonal is satisfied provided that its initial value satisfies this constraint. Local existence and uniqueness of solutions to the initial boundary value problem of the resulting viscoelastic fluid system are established. It is also shown that the local solutions can be extended globally provided that the data are small enough.
\end{abstract}

Keywords: Intermediate configuration; intermediate tensor; isochoric.

\section{Introduction}

A significant number of fluids, both natural and manufactured, may be modeled as viscoelastic. Blood is a key example of a naturally occurring viscoelastic fluid, ${ }^{1}$ while a range of polymeric fluids generally fall into this class. ${ }^{2}$

An important class of viscoelastic fluids is those of differential type, with the variables of interest being the velocity, pressure, and extra stress. The resulting set 
of nonlinear equations generally has the features of a mixed parabolic-hyperbolic system. The problem studied in this work falls within this class.

Not surprisingly, problems involving viscoelastic fluids offer significant theoretical and computational challenges. With regard to theoretical studies, the work by Guillopé and Saut ${ }^{9}$ represents an early, important contribution. These authors established existence locally and globally in time, and uniqueness of solutions, for the equations corresponding to an Oldroyd-B fluid. Chemin and Masmoudi ${ }^{5}$ established the global existence for small data to the Cauchy problem for a fluid flow with a differential law of Oldroyd-B type making use of Besov theory. The approach pioneered by Lions for compressible Navier-Stokes equation to define renormalized solutions has been adapted by Lions and Masmoudi ${ }^{19}$ to prove the existence of global weak solutions with general initial data. A key result in that approach, after obtaining some uniform a priori estimates, is that in some sense compactness in $L^{2}$ for the extra stress is propagated in time by the transport equation. On the other hand, Sideris and Thomases ${ }^{28}$ studied the Cauchy problem for incompressible nonlinear isotropic elastodynamics making use of the Galilean invariance for such a system, a weighted $L^{2}$ estimate, and the null condition. They obtained the global-in-time existence of classical solutions and decay for small initial displacements. Since all the obtained are uniform with respect to the viscosity, the incompressible limit of the isotropic elastodynamics is also examined. Finally Fanghua Lin and Ping Zhang ${ }^{16}$ have obtained global-in-time existence of solutions for an Oldroyd model by adapting the hyperbolic type approach, provided that the initial data are close enough to the equilibrium state. It should be mentioned that all these models involve nonlocal (in time) constitutive equations. There are also those models where the relations between the stress and strain can be expressed in local form. An interesting and related work is that by Lin and $\mathrm{Liu}^{15}$ on the dynamics of systems which model the flow of liquid crystals. One of the features of this work is the presence of an algebraic constraint that must be satisfied at each time.

Neças and co-author, have studied the flow of fluids where the viscosity is a function on both the pressure $p$ and the symmetric part of the rate of the deformation gradient $\mathbf{D} .^{20,21}$ They have established the existence of weak solutions for spatially periodic three-dimensional flows that are global-in-time, for a large class of physically meaningful viscosity-pressure relationships. Their method of proof also provides the existence of a strong solution for a short time interval or a global strong solution for small data that are unique in the class of weak solutions. It is worth noting that the pressure is determined up to a function of time.

As far as computational studies for viscoelastic fluids are concerned, a comprehensive overview may be found in the monograph by Owens and Philips. ${ }^{24}$

It is well known that a viscoelastic material can be regarded as a transition state between fluid and solid. A particularly interesting class of viscoelastic fluids is those that are modeled as continua with multiple natural configurations or 
stress-free states. Materials which fall within this class include metal plasticity, viscoelastic liquids, anisotropic fluids, to mention a few. Many complex hydrodynamic and rheological features of complex fluids can be viewed as consequences of an internal elastic response. Rao and Rajagopal ${ }^{27}$ have developed a continuum thermodynamic framework for materials that have an elastic response in an evolving set of natural configurations, making use among others of the Helmholtz potential. ${ }^{a}$ In related work, Rajagopal and co-workers ${ }^{12,13}$ have used this framework to model polymers which undergo a phase transition from the melt phase to one of flow-induced crystallization, and finally to a solid state. More recently, Karra and Rajagopal ${ }^{14}$ have extended the framework presented in Ref. 27 by allowing the intermediate configuration to evolve without an instantaneous elastic response. The qualitative properties of the problems described have not been studied in any detail, and in this, the first of a series of papers, we shall investigate the model where only an elastic response is allowed in the intermediate configuration. The focus in this work is on the melt phase, and the model is that developed and presented by Kannan, Rao and Rajagopal. ${ }^{12}$ The novelty of the problem, from the mathematical point of view, derives from various features: the intermediate deformation tensor which represents the reference state, and its evolution; the constraint that these tensors be proper orthogonal, that is, that they have a determinant equal to unity; the need to take account of thermal effects, and to include temperature as a variable; and finally, the highly coupled and nonlinear nature of the problem.

The aim of this work is to establish local and global existence in time of solutions, and uniqueness of solutions, for the melt problem with a single relaxation time; the extension to multiple relaxation times may be readily made. The approach pioneered by Guillopé and Saut ${ }^{9}$ is adopted, in which local existence of solutions are shown through the use of a fixed-point method, while global existence of solutions are obtained by first obtaining uniform a priori bounds of the solutions constructed locally, using once more the arguments presented in Ref. 9. Note that the question of defining weak solutions in the sense of either Ref. 19 or 20 for the problem under investigation is open and seems complicated due to the strong coupling, the nonlinearity of the system, and the nontrivial transport equation associated with the intermediate deformation tensor $\mathbf{B}$.

The structure of the rest of this work is as follows. In Sec. 2, the initial-boundaryvalue problem is formulated. Then, in Sec. 3, it is shown that the intermediate deformation tensor $\mathbf{B}$ satisfies the constraint $\operatorname{det} \mathbf{B}=1$ if and only if this condition is satisfied initially. In Sec. 4, various results for function spaces are presented, and estimates for some problems related to the original initial-value problem are derived. These estimates are essential for establishing solvability of the problem using a Leray-Schauder fixed point argument, which is presented in Sec. 5. Finally, the local solution obtained in Sec. 5, is extended globally in time in Sec. 6 .

${ }^{a}$ More recently, Rajagopal and Srinivasa ${ }^{26}$ have developed a class of implicit models for viscoelastic materials based on the Gibbs potential. 


\section{The Initial-Boundary-Value Problem for the Polymer Melt}

\subsection{Governing equations}

The essential features of the melt model are presented for the case of a single relaxation time, based on Refs. 12 and 27. The extension to multiple relaxation times may be carried out readily.

A fluid occupies a bounded domain $\Omega \subset \mathbb{R}^{d}(d=2,3)$ with boundary $\Gamma$, and is subject to the action of a body force $\mathbf{b}$ per unit mass. The mass density of the fluid is assumed, without loss of generality, to be equal to unity. It is required to find the velocity $\mathbf{u}(\mathbf{x}, t)$, the pressure $p(\mathbf{x}, t)$, the temperature $\theta(\mathbf{x}, t)$, and the "intermediate" left Cauchy-Green tensor B (which contains the information about the orientation of the molecules in the melt) which satisfy the following set of equations: conservation of momentum

$$
\mathbf{u}_{t}+[\mathbf{u} \cdot \nabla] \mathbf{u}-\operatorname{div} \mathbf{T}=\mathbf{b}
$$

conservation of mass (continuity equation)

$$
\operatorname{div} \mathbf{u}=0
$$

conservation of energy

$$
\theta_{t}+[\mathbf{u} \cdot \nabla] \theta+\operatorname{div} \mathbf{q}=\mathbf{T}: \mathbf{D}+r
$$

where $r$ is the radiant heating, the heat flux $\mathbf{q}$ is given by Fourier's law

$$
\mathbf{q}=-\kappa \nabla \theta
$$

with $\kappa$ the thermal conductivity, and the heat capacity has been set equal to unity in (2.3). The constitutive equation for the stress is given by

$$
\mathbf{T}=-p I+\alpha_{1} \theta \mathbf{B}+2 \nu \mathbf{D}
$$

where $\alpha_{1}$ is a positive constant, $\mathbf{I}$ is the identity tensor, and $\mathbf{D}$ is the rate of deformation tensor which is defined by

$$
\mathbf{D}=\frac{1}{2}\left[\nabla \mathbf{u}+(\nabla \mathbf{u})^{\mathrm{T}}\right]
$$

Here and henceforth $(\cdot)_{t} \equiv \partial_{t}$ denotes the partial derivative with respect to time.

The motion of the fluid is represented by the function $\mathrm{x}=\boldsymbol{\varphi}(\mathrm{X}, t)$ in which $\mathbf{X}$ and $\mathbf{x}$ are respectively the initial and current positions of a material point in the body. The deformation gradient associated with this motion is defined by $\mathbf{F}=\nabla_{X} \boldsymbol{\varphi}$.

The intermediate deformation tensor $\mathbf{F}_{I}$ gives the deformation between the reference and intermediate configurations, and is required to be non-singular. The 
corresponding symmetric intermediate left Cauchy-Green tensor $\mathbf{B}$ is defined by ${ }^{\mathrm{b}}$

$$
\mathbf{B}=\mathbf{F}_{I} \mathbf{F}_{I}^{\mathrm{T}} \text {. }
$$

At this stage it should be pointed out that $\mathbf{F}_{I}$ is not in general the gradient of a mapping. ${ }^{25}$

The evolution equation for the tensor $\mathbf{B}$ is given by

$$
\frac{D \mathbf{B}}{D t}-[\nabla \mathbf{u}] \mathbf{B}-\mathbf{B}[\nabla \mathbf{u}]^{\mathrm{T}}=\alpha_{2} \theta\left[\frac{d}{\operatorname{tr} \mathbf{B}^{-1}} \mathbf{I}-\mathbf{B}\right],
$$

where $\alpha_{2}$ stands for a positive constant, and $D \mathbf{B} / D t$ is the material time derivative of $\mathbf{B}$, that is,

$$
\frac{D \mathbf{B}}{D t}=\mathbf{B}_{t}+[\mathbf{u} \cdot \nabla] \mathbf{B}
$$

Note that $\mathbf{B}$ is non-singular so that $\mathbf{B}^{-1}$ exists, by virtue of $\mathbf{F}_{I}$ being non-singular.

Thus, (2.6) can be written as

$$
\mathbf{B}_{t}=-[\mathbf{u} \cdot \nabla] \mathbf{B}+[\nabla \mathbf{u}] \mathbf{B}+\mathbf{B}[\nabla \mathbf{u}]^{\mathrm{T}}+\alpha_{2} \theta\left[\frac{d}{\operatorname{tr} \mathbf{B}^{-1}} \mathbf{I}-\mathbf{B}\right] .
$$

As the fluid is incompressible, it is required in addition that the motion associated with the intermediate configuration be isochoric; that is,

$$
\operatorname{det} \mathbf{B}=1 .
$$

After substitution of the constitutive equations for the stress $\mathbf{T}$ in (2.1) and the heat flux $\mathbf{q}$ in (2.3), the initial-boundary-value problem for the melt may be summarized as follows: find $(\mathbf{u}, p, \mathbf{B}, \theta)$ such that for $(t, \mathbf{x}) \in(0, T) \times \Omega$,

$$
\left\{\begin{array}{l}
\mathbf{u}_{t}+[\mathbf{u} \cdot \nabla] \mathbf{u}-\nu \Delta \mathbf{u}-\alpha_{1}(\mathbf{B} \nabla \theta+\theta \operatorname{div} \mathbf{B})+\nabla p=\mathbf{b}, \\
\mathbf{B}_{t}+[\mathbf{u} \cdot \nabla] \mathbf{B}-[\nabla \mathbf{u}] \mathbf{B}-\mathbf{B}[\nabla \mathbf{u}]^{\mathrm{T}}=\alpha_{2} \theta\left[\frac{d}{\operatorname{tr} \mathbf{B}^{-1}} \mathbf{I}-\mathbf{B}\right], \\
\theta_{t}+[\mathbf{u} \cdot \nabla] \theta-\kappa \Delta \theta=\left[\alpha_{1} \theta \mathbf{B}+2 \nu \mathbf{D}\right]: \mathbf{D}+r, \\
\operatorname{div} \mathbf{u}=0, \operatorname{det} \mathbf{B}=1, \mathbf{B}=\mathbf{B}^{\mathrm{T}},
\end{array}\right.
$$

together with the boundary conditions

$$
\left.\theta\right|_{\partial \Omega}=0 \quad \text { and }\left.\quad \mathbf{u}\right|_{\partial \Omega}=0
$$

and initial conditions

$$
\theta(\mathbf{x}, 0)=\theta_{0}, \quad \mathbf{u}(\mathbf{x}, 0)=\mathbf{u}_{0} \quad \text { and } \quad \mathbf{B}(\mathbf{x}, \mathbf{0})=\mathbf{B}_{0},
$$

where of course, we have the compatibility condition

$$
\operatorname{div} \mathbf{u}_{0}=0 .
$$

b The notation $\mathbf{B}$ conventionally denotes the left Cauchy-Green tensor $\mathbf{F F}^{\mathrm{T}}$ in continuum mechanics. This tensor plays no role in the problem discussed here, so the notation $\mathbf{B}$ (with a subscript, for convenience) is used for the intermediate tensor without danger of confusion. 
We have chosen to work with homogeneous condition on the velocity in order to avoid the technical arguments linked to the Hopf lemma (see Ref. 8, Chap. 4, Lemma 2.3).

Remarks. (a) The more relevant physical condition $\left.\theta\right|_{\partial \Omega}=\theta_{b}$ may be adopted instead of $(2.10)_{1}$, but with the help of the lifting operator $\mathcal{L}$, that is, an operator from $H^{1 / 2}(\partial \Omega)$ into $H^{1}(\Omega)$ which is continuous from $H^{s+1 / 2}(\partial \Omega)$ into $H^{s+1}(\Omega)$ for all $s \geq 0$ (the existence of such an operator is established in Ref. 8 for instance), it is always possible to revert to the homogeneous boundary condition. Indeed, considering $\theta_{b}(t) \in H^{1 / 2}(\partial \Omega)$ (at least), we denote by $\widetilde{\theta}$ the function defined for a.e. $t, 0<t<T$, by

$$
\widetilde{\theta}(t)=\mathcal{L} \theta_{b}(t) .
$$

It is manifest that the function $\widetilde{\theta}$ is an element of $L^{2}\left(0, T ; H^{1}(\Omega)\right)$ and one has

$$
\|\widetilde{\theta}\|_{L^{2}\left(0, T ; H^{1}(\Omega)\right.} \leq C\left\|\theta_{b}\right\|_{L^{2}\left(0, T ; H^{1 / 2}(\partial \Omega)\right.} .
$$

When setting $\theta^{*}=\theta-\widetilde{\theta}$, one sees that $\left.\theta^{*}\right|_{\partial \Omega}=0$. There is thus no loss of generality in considering a homogeneous boundary condition.

(b) In the problem described by (2.9)-(2.11), the incompressibility condition $\operatorname{det} \mathbf{B}=1$ is not linked to the incompressibility of the fluid $\operatorname{div} \mathbf{u}=0$, while for the model of viscoelastic fluid discussed in Ref. 17, both conditions are the same because the deformation of the material is described by the tensor $\mathbf{F}=\partial \mathbf{x} / \partial \mathbf{X}$, where $\mathbf{x}(t, \mathbf{X})$ is the flow map. Since

$$
\frac{D}{D t} \operatorname{det} \mathbf{F}=\operatorname{det} \mathbf{F} \operatorname{div} \mathbf{u},
$$

one sees that $\operatorname{div} \mathbf{u}=0$ is equivalent to $\operatorname{det} \mathbf{F}=1$.

(c) The first equation in the system of Eqs. (2.9), is essentially an extension of the Navier-Stokes equation, while the third one is an extension of the standard equation for heat convection and conduction, in both cases with the addition of terms which capture the coupling with temperature and velocity, respectively, and the state of the fluid in the intermediate configuration.

\section{Some Properties of $B$}

\subsection{The constraint $\operatorname{det} \mathrm{B}=1$}

The presence of the nonlinear constraint $\operatorname{det} \mathbf{B}=1$ is a complicating factor in determining the solvability of $(2.9)-(2.11)$. We show that the constraint can be removed provided that the initial condition $\operatorname{det} \mathbf{B}(\mathbf{x}, 0)=1$ is imposed. Indeed, setting

$$
J=\operatorname{det} \mathbf{B}
$$


and from a general result on derivatives of determinants (see Ref. 4, p. 20) and using $(2.9)_{2}$ we have, with a superposed dot denoting the material derivative $D / D t$,

$$
\begin{aligned}
J^{-1} \dot{J} & =\mathbf{B}^{-\mathrm{T}}: \dot{\mathbf{B}} \\
& =\mathbf{B}^{-\mathrm{T}}:\left([\nabla \mathbf{u}] \mathbf{B}+\mathbf{B}[\nabla \mathbf{u}]^{\mathrm{T}}+\alpha_{2} \theta\left[\frac{d}{\operatorname{tr} \mathbf{B}^{-1}} I-\mathbf{B}\right]\right) \\
& =2 \operatorname{div} \mathbf{u}+\alpha_{2} \theta\left[\frac{d}{\operatorname{tr} \mathbf{B}^{-1}} I-\mathbf{B}\right]: \mathbf{B}^{-\mathrm{T}} \\
& =2 \operatorname{div} \mathbf{u}+\alpha_{2} \theta\left[\frac{d \operatorname{tr}\left(\mathbf{B}^{-\mathrm{T}}\right)}{\operatorname{tr} \mathbf{B}^{-1}}-\operatorname{tr}\left(\mathbf{B}^{-1} \mathbf{B}\right)\right] \\
& =2 \operatorname{div} \mathbf{u} .
\end{aligned}
$$

For an incompressible fluid it follows that $\dot{J}=0$, which implies that

$$
J=\operatorname{det} \mathbf{B}=\operatorname{det} \mathbf{B}_{0} .
$$

Thus we have the following result.

Lemma 3.1. Let $\mathbf{B}$ be the intermediate left Cauchy-Green tensor satisfying $(2.9)_{2}$, and being symmetric. Then $\operatorname{det} \mathbf{B}(t)=1$ if and only if $\operatorname{det} \mathbf{B}(0)=1$.

In conclusion, we have shown that

Lemma 3.2. The set $(\mathbf{u}, p, \mathbf{B}, \theta)$ is a solution of (2.9)-(2.11) if and only if it solves

$$
\left\{\begin{array}{l}
\mathbf{u}_{t}+[\mathbf{u} \cdot \nabla] \mathbf{u}-\nu \Delta \mathbf{u}-\alpha_{1}(\mathbf{B} \nabla \theta+\theta \operatorname{div} \mathbf{B})+\nabla p=\mathbf{b}, \\
\mathbf{B}_{t}+[\mathbf{u} \cdot \nabla] \mathbf{B}-[\nabla \mathbf{u}] \mathbf{B}-\mathbf{B}[\nabla \mathbf{u}]^{\mathrm{T}}=\alpha_{2} \theta\left[\frac{d}{\operatorname{tr} \mathbf{B}^{-1}} I-\mathbf{B}\right], \\
\theta_{t}+[\mathbf{u} \cdot \nabla] \theta-\kappa \Delta \theta=\left[\alpha_{1} \theta \mathbf{B}+2 \nu \mathbf{D}\right]: \mathbf{D}+r, \\
\operatorname{div} \mathbf{u}=0, \\
\left.\theta\right|_{\partial \Omega}=0, \quad \theta(\mathbf{x}, 0)=\theta_{0},\left.\quad \mathbf{u}\right|_{\partial \Omega}=0, \quad \mathbf{u}(\mathbf{x}, 0)=\mathbf{u}_{0}, \\
\mathbf{B}=\mathbf{B}^{\mathrm{T}}, \quad \mathbf{B}(\mathbf{x}, \mathbf{0})=\mathbf{B}_{0}, \quad \operatorname{det} \mathbf{B}_{0}=1 .
\end{array}\right.
$$

\section{Auxiliary Results}

For a multi-index $\alpha=\left(\alpha_{1}, \ldots, \alpha_{d}\right)$ where $\alpha_{i}$ are non-negative integers, we define

$$
\partial^{\alpha}(\cdot):=\frac{\partial^{\alpha_{1}+\cdots+\alpha_{d}}(\cdot)}{\partial^{\alpha_{1}} x_{1} \cdots \partial^{\alpha_{d}} x_{d}} .
$$

Leibniz's formula can be expressed using multi-index notation in the form as in Ref. 6,

$$
\partial^{\alpha}(u v)=\sum_{\beta_{i} \leq \alpha_{i}}\left(\begin{array}{l}
\alpha \\
\beta
\end{array}\right) \partial^{\beta} u \partial^{\alpha-\beta} v
$$


where

$$
\left(\begin{array}{l}
\alpha \\
\beta
\end{array}\right)=\frac{\alpha !}{\beta !(\alpha-\beta) !}
$$

and $\alpha !=\alpha_{1} ! \cdots \alpha_{d} !$.

We introduce the classical function spaces

$$
\begin{aligned}
M:=H_{0}^{1}(\Omega)^{d} & =\text { closure of } C_{0}^{\infty}\left(\Omega, \mathbb{R}^{d}\right) \text { in the norm }\left(\int_{\Omega}|\nabla \mathbf{v}|^{2} \mathrm{dx}\right)^{1 / 2}, \\
H^{2}(\Omega)^{d} & =\left\{\mathbf{v} \in L^{2}\left(\Omega, \mathbb{R}^{d}\right): \partial^{\beta} \mathbf{v} \in L^{2}\left(\Omega, \mathbb{R}^{d}\right),|\beta| \leq 2\right\} \\
W & =C_{0}^{\infty}\left(\Omega, \mathbb{R}^{d}\right) \cap\{\mathbf{v}: \operatorname{div} \mathbf{v}=0\} \\
V & =\text { closure of } W \text { in } H^{1}(\Omega) \\
H & =\text { closure of } W \text { in } L^{2}\left(\Omega, \mathbb{R}^{d}\right), \\
H^{-1}(\Omega) & =\text { the dual of } H_{0}^{1}(\Omega) \\
X & =\left\{\mathbf{B}=\left(B_{i j}\right) \mid B_{i j} \in L^{2}(\Omega), B_{i j}=B_{j i}, 1 \leq i, j \leq d\right\}
\end{aligned}
$$

Clearly, from the identification of $H$ with its dual, one has the triplet $V \subset H \subset V^{\prime}$. We will denote by $\|\cdot\|_{k}$ the classical Sobolev norm, and by $(\cdot, \cdot)$ and $\|\cdot\|$ the $L^{2}$-inner product and norm respectively. The notation will be used for scalar-, vector- and matrix-valued functions, with the norms for the latter two types of functions being defined in the usual, componentwise way. As usual, $\mathbf{u}(t)$ stands for the function $\mathbf{x} \in \Omega \mapsto \mathbf{u}(\mathbf{x}, t)$.

If $I$ is an interval of $\mathbb{R}_{+}$, we denote by $\mathcal{C}(\bar{I}, V)$ the space of vector-valued functions $\mathbf{v}(t, \mathbf{x})$ such that $\mathbf{v}(t) \in V$ for all $t \in \bar{I}$ and the function $t \mapsto \mathbf{v}(t, \cdot)$ with values in $V$ is continuous on $\bar{I}$. $\mathcal{C}_{b}(I, V)$ is the space of bounded continuous functions on $\bar{I}$ into $V$. Finally, if $E$ is a Banach space, $L^{p}(I, E)$ for $1 \leq p \leq \infty$ consists of $p$-integrable functions with values in $E$.

In inequalities that will occur later, $C$ denotes a generic positive constant which may take different values even in the same calculation. The entities on which it may depend, are given in brackets, e.g. $C(\Omega)$ denotes a constant which depends at most on $\Omega$.

We denote by $\mathcal{P}$ the orthogonal projection of $L^{2}(\Omega)^{d}$ onto $H$, and by $D(\mathbb{A})$ the domain of the Stokes operator $\mathbb{A}=-\nu \mathcal{P} \Delta$. It can be shown (see Ref. 30) that

$$
D(\mathbb{A})=H^{2}(\Omega)^{d} \cap V,
$$

and that there exists a positive constant $C$ such that

$$
\|\mathbf{u}\|_{2} \leq C\|\mathbb{A} \mathbf{u}\| \quad \text { for all } \mathbf{u} \in D(\mathbb{A}) .
$$


We will make reference to the following results.

Lemma 4.1. (Ref. 30) Let $\Omega$ be a bounded open set of $\mathbb{R}^{d}$, with boundary $\partial \Omega$ of class $C^{3}$. If $\mathbf{v}_{0} \in D(\mathbb{A}), \mathbf{F} \in L^{2}\left(0, T ; H^{1}(\Omega)^{d}\right)$ and $\mathbf{F}_{t} \in L^{2}\left(0, T ; H^{-1}(\Omega)^{d}\right)$, then the time-dependent Stokes problem

$$
\left\{\begin{array}{l}
\mathbf{v}_{t}+\mathbb{A} \mathbf{v}=\mathbf{F}, \quad \text { a.a. } t \in(0, T) \\
\mathbf{v}(0)=\mathbf{v}_{0},
\end{array}\right.
$$

admits a unique solution $\mathbf{v}(t)$ and associated pressure $p(t)$, with

$$
\begin{aligned}
& \mathbf{v} \in L^{2}\left(0, T ; H^{3}(\Omega)^{d} \cap V\right) \cap \mathcal{C}(0, T ; D(\mathbb{A})), \quad \mathbf{v}_{t} \in L^{2}(0, T ; V) \cap \mathcal{C}(0, T ; H), \\
& \nabla p \in L^{2}\left(0, T ; H^{1}(\Omega)\right) .
\end{aligned}
$$

Moreover, there exists a positive constant $C$ such that

$$
\begin{gathered}
\|\mathbf{v}\|_{L^{2}\left(0, T ; H^{3}\right) \cap L^{\infty}(0, T ; D(\mathbb{A}))}^{2}+\left\|\mathbf{v}_{t}\right\|_{L^{2}(0, T ; V) \cap L^{\infty}(0, T ; H)}^{2}+\|p\|_{L^{2}\left(0, T ; H^{2}\right)}^{2} \\
\leq C\left[\left\|\mathbb{A} \mathbf{v}_{0}\right\|^{2}+\|\mathbf{F}\|_{L^{2}\left(0, T ; H^{1}\right)}^{2}+\left\|\mathbf{F}_{t}\right\|_{L^{2}\left(0, T ; H^{-1}\right)}^{2}+\|\mathbf{F}(\mathbf{0})\|^{2}\right] .
\end{gathered}
$$

Next, we recall the following result for the time-dependent heat equation.

Lemma 4.2. (Ref. 6, pp. 360-361) Let $\Omega$ be a bounded open set of $\mathbb{R}^{d}$, with a smooth boundary $\partial \Omega$. If $\theta_{0} \in H_{0}^{1}(\Omega)$ and $f \in L^{2}\left(0, T ; L^{2}(\Omega)\right)$, then the problem

$$
\left\{\begin{array}{l}
\theta_{t}-\kappa \Delta \theta=f, \quad \text { a.a. } t \in(0, T), \\
\theta(0)=\theta_{0}, \\
\left.\theta\right|_{\partial \Omega}=0, \quad \text { a.a. } t \in(0, T),
\end{array}\right.
$$

admits a unique solution $\theta(t)$, with

$$
\theta \in L^{2}\left(0, T ; H^{2}(\Omega)\right) \cap L^{\infty}\left(0, T ; H_{0}^{1}(\Omega)\right), \quad \theta_{t} \in L^{2}\left(0, T ; L^{2}(\Omega)\right) .
$$

Furthermore, there exists a positive constant $C \equiv C(\kappa, \Omega)$, such that

$$
\|\theta\|_{L^{\infty}\left(0, T ; H^{1}\right)}+\|\theta\|_{L^{2}\left(0, T ; H^{2}\right)}+\left\|\theta_{t}\right\|_{L^{2}\left(0, T ; L^{2}(\Omega)\right)} \leq C\left[\|f\|_{L^{2}\left(0, T ; L^{2}(\Omega)\right)}+\left\|\theta_{0}\right\|_{1}\right] .
$$

If in addition $\theta_{0} \in H^{2}(\Omega)$ and $f_{t} \in L^{2}\left(0, T ; L^{2}(\Omega)\right)$, then

$$
\begin{gathered}
\theta \in L^{\infty}\left(0, T ; H^{2}(\Omega)\right), \quad \theta_{t} \in L^{\infty}\left(0, T ; L^{2}(\Omega)\right) \cap L^{2}\left(0, T ; H_{0}^{1}(\Omega)\right), \\
\theta_{t t} \in L^{2}\left(0, T ; H^{-1}(\Omega)\right),
\end{gathered}
$$

and there exists a positive constant $C \equiv C(\kappa, \Omega)$, such that

$$
\begin{aligned}
& \|\theta\|_{L^{\infty}\left(0, T ; H^{2}\right)}^{2}+\left\|\theta_{t}\right\|_{L^{\infty}\left(0, T ; L^{2}\right) \cap L^{2}\left(0, T ; H^{1}\right)}^{2}+\left\|\theta_{t t}\right\|_{L^{2}\left(0, T ; H^{-1}(\Omega)\right)}^{2} \\
& \quad \leq C\left[\|f\|_{H^{1}\left(0, T ; L^{2}(\Omega)\right)}^{2}+\left\|\theta_{0}\right\|_{2}^{2}\right] .
\end{aligned}
$$


In order to analyze the transport equation in (3.3), we let

$$
\widetilde{\mathbf{u}}(t) \in D(\mathbb{A}) \cap H^{3}(\Omega)^{d} \text { and } \tilde{\theta}(t) \in H_{0}^{1}(\Omega) \cap H^{2}(\Omega) .
$$

Now, we introduce the following problem: find $\mathbf{B}(t) \in X$ such that

$$
\left\{\begin{array}{l}
\mathbf{B}_{t}+[\widetilde{\mathbf{u}} \cdot \nabla] \mathbf{B}=[\nabla \widetilde{\mathbf{u}}] \mathbf{B}+\mathbf{B}[\nabla \widetilde{\mathbf{u}}]^{\mathrm{T}}+\alpha_{2} \tilde{\theta}\left[\frac{d}{\operatorname{tr} \mathbf{B}^{-1}} \mathbf{I}-\mathbf{B}\right], \quad \text { a.a. } t \in(0, T) \\
\mathbf{B}(\mathbf{0})=\mathbf{B}_{0} .
\end{array}\right.
$$

We first investigate some qualitative properties associated with the solution of problem (4.13). We then claim the following:

Lemma 4.3. Let $(\widetilde{\mathbf{u}}(t), \widetilde{\theta}(t)) \in D(\mathbb{A}) \cap H^{3}(\Omega)^{d} \times H_{0}^{1}(\Omega) \cap H^{2}(\Omega)$. Let $\mathbf{B}$ be a regular solution to the system (4.13). If $\mathbf{B}_{0}$ is symmetric and $\operatorname{det} \mathbf{B}_{0}=1$, then the solution $\mathbf{B}$ remains so for all later times of existence of solutions.

Proof. Following Lemma 3.1, we readily see that $\operatorname{det} \mathbf{B}=1$ for all later times of existence of solutions if $\operatorname{det} \mathbf{B}_{0}=1$.

Next, in order to show that $\mathbf{B}$ remains symmetric, we follow Ref. 7, and let

$$
\mathbf{H}=\mathbf{B}-\mathbf{B}^{\mathrm{T}}, \quad \mathbf{K}=\mathbf{B}^{-1}-\mathbf{B}^{-\mathrm{T}} .
$$

One has $\operatorname{tr} \mathbf{K}=0=\operatorname{tr} \mathbf{H}, \operatorname{tr} \mathbf{B}=\operatorname{tr} \mathbf{B}^{\mathrm{T}}$, and $\operatorname{tr} \mathbf{B}^{-1}=\operatorname{tr} \mathbf{B}^{-\mathrm{T}}$, since $\mathbf{K}$ and $\mathbf{H}$ are skew-symmetric. From (4.13), one obtains

$$
\left\{\begin{array}{l}
\mathbf{H}_{t}=-[\widetilde{\mathbf{u}} \cdot \nabla] \mathbf{H}+[\nabla \widetilde{\mathbf{u}}] \mathbf{H}+\mathbf{H}[\nabla \widetilde{\mathbf{u}}]^{\mathrm{T}}-\alpha_{2} \widetilde{\theta} \mathbf{H} \\
\mathbf{H}(\mathbf{0})=\mathbf{H}_{0}
\end{array}\right.
$$

A standard energy estimate gives

$$
\begin{aligned}
\frac{1}{2} \frac{d}{d t}\|\mathbf{H}(t)\|^{2} & =\int_{\Omega}[\nabla \widetilde{\mathbf{u}}] \mathbf{H}: \mathbf{H}+\int_{\Omega} \mathbf{H}[\nabla \widetilde{\mathbf{u}}]^{\mathrm{T}}: \mathbf{H}-\alpha_{2} \int_{\Omega} \widetilde{\theta} \mathbf{H}: \mathbf{H} \\
& =-2 \int_{\Omega}[\nabla \widetilde{\mathbf{u}}]: \mathbf{H}^{2}-\alpha_{2} \int_{\Omega} \widetilde{\theta} \mathbf{H}: \mathbf{H} \\
& \leq C\left(\|\widetilde{\mathbf{u}}(t)\|_{3}+\|\widetilde{\theta}(t)\|_{2}\right)\|\mathbf{H}\|^{2},
\end{aligned}
$$

which by Gronwall's lemma and the assumptions made on $\widetilde{\mathbf{u}}$ and $\widetilde{\theta}$ leads to $\mathbf{H}(t)=\mathbf{0}$; that is, $\mathbf{B}=\mathbf{B}^{\mathrm{T}}$.

As far as the existence of solutions of (4.13) is concerned, we quickly observe that we are dealing with a first-order nonlinear hyperbolic system of equations. Thus, following the method of characteristics (see Ref. 11, p. 15), (4.13) can be transformed into a nonlinear first-order system of ordinary differential equations in a Banach space $X$. Consequently, its existence relies on the application of the Cauchy-Lipschitz theorem (see Ref. 10, p. 60).

So, we need to show that the nonlinear mapping defined by

$$
\mathbf{B} \mapsto F(\mathbf{B})=[\nabla \widetilde{\mathbf{u}}] \mathbf{B}+\mathbf{B}[\nabla \widetilde{\mathbf{u}}]^{\mathrm{T}}+\alpha_{2} \widetilde{\theta}\left[\frac{d}{\operatorname{tr} \mathbf{B}^{-1}} \mathbf{I}-\mathbf{B}\right]
$$

is Lipschitz continuous on $X$. 
We recall that the symbol $|\cdot|$ will denote the absolute value in the case of scalars, the Euclidean norm $|\mathbf{u}|^{2}=\mathbf{u} \cdot \mathbf{u}$ for vectors, and the Frobenius norm $|\mathbf{A}|^{2}=$ $\sum_{i, j=1}^{d} A_{i j} A_{i j}$ in the case of tensors or matrices. We also mention that if $\mathbf{A}$ and $\mathbf{B}$ are two invertible matrices, then the following identity holds:

$$
\mathbf{A}^{-1}-\mathbf{B}^{-1}=\mathbf{A}^{-1}(\mathbf{B}-\mathbf{A}) \mathbf{B}^{-1} .
$$

Now, to show that $F$ is Lipschitz continuous on $X$, let $\mathbf{B}, \mathbf{A} \in X$, then from (4.15) and triangle inequality

$$
\begin{aligned}
|F(\mathbf{B})-F(\mathbf{A})| & \leq 2|\nabla \widetilde{\mathbf{u}}||\mathbf{B}-\mathbf{A}|+\alpha_{2}|\widetilde{\theta}(\mathbf{B}-\mathbf{A})|+d^{2} \alpha_{2}|\widetilde{\theta}|\left|\frac{\operatorname{tr}\left(\mathbf{A}^{-1}(\mathbf{B}-\mathbf{A}) \mathbf{B}^{-1}\right)}{\operatorname{tr} \mathbf{B}^{-1} \operatorname{tr} \mathbf{A}^{-1}}\right| \\
& \leq\left[2|\nabla \widetilde{\mathbf{u}}|+\alpha_{2}|\widetilde{\theta}|+d^{2} \alpha_{2}|\widetilde{\theta}| \frac{\left|\mathbf{A}^{-1}\right|\left|\mathbf{B}^{-1}\right|}{\left|\operatorname{tr} \mathbf{A}^{-1}\right|\left|\operatorname{tr} \mathbf{B}^{-1}\right|}\right]|\mathbf{B}-\mathbf{A}| \\
& \leq\left[2|\nabla \widetilde{\mathbf{u}}|+\alpha_{2}\left(1+d^{2}\right)|\widetilde{\theta}|\right]|\mathbf{B}-\mathbf{A}|,
\end{aligned}
$$

from which we easily get

$$
\|F(\mathbf{B})-F(\mathbf{A})\| \leq C\left(\|\widetilde{\mathbf{u}}\|_{3}+\|\widetilde{\theta}\|_{2}\right)\|\mathbf{B}-\mathbf{A}\|,
$$

where we have used Agmon's inequalities ${ }^{31}$ (p. 52):

$$
\|u\|_{L^{\infty}(\Omega)} \leq\left\{\begin{array}{lll}
C\|u\|_{H^{d / 2-1}(\Omega)}^{1 / 2}\|u\|_{H^{d / 2+1}(\Omega)}^{1 / 2} & \forall u \in H^{d / 2+1}(\Omega) & \text { if } d \text { is even, } \\
C\|u\|_{H^{(d-1) / 2}(\Omega)}^{1 / 2}\|u\|_{H^{(d+1) / 2}(\Omega)}^{1 / 2} & \forall u \in H^{(d+1) / 2}(\Omega) & \text { if } d \text { is odd. }
\end{array}\right.
$$

We conclude that $F(\mathbf{B})$ is Lipschitz continuous on $X$.

Of course more regular solutions of (4.13) can be obtained by imposing stronger regularity conditions on the initial data $\mathbf{B}_{0}$. Indeed one can claim the following result.

Lemma 4.4. Let $\Omega$ be a regular domain of $\mathbb{R}^{d}$ with boundary $\partial \Omega$ of class $C^{1}$. Assume that $\mathbf{B}_{0} \in X \cap H^{2}(\Omega)^{d \times d}$. Then there exists a positive constant $T$ such that for $\widetilde{\mathbf{u}} \in L^{\infty}\left(0, T ; H^{3}(\Omega)^{d} \cap D(\mathbb{A})\right)$ and $\widetilde{\theta} \in L^{\infty}\left(0, T ; H^{2}(\Omega) \cap H_{0}^{1}(\Omega)\right)$, the solution of (4.13) enjoy the following regularity:

$$
\mathbf{B} \in L^{\infty}\left(0, T ; H^{2}(\Omega)^{d \times d} \cap X\right) \quad \text { and } \quad \mathbf{B}_{t} \in L^{\infty}\left(0, T ; L^{2}(\Omega)^{d \times d}\right) .
$$

Moreover, if

$$
C\left(\|\widetilde{\mathbf{u}}\|_{L^{1}\left(0, T ; H^{3}\right)}+\|\widetilde{\theta}\|_{L^{1}\left(0, T ; H^{2}\right)}+\|\widetilde{\theta}\|_{L^{2}\left(0, T ; H^{2}\right)}^{2}\right) \leq \frac{1}{\left\|\mathbf{B}_{0}\right\|_{2}^{4}},
$$

then

$$
\|\mathbf{B}(t)\|_{2} \leq\left\|\mathbf{B}_{0}\right\|_{2} \quad \text { for } t \in[0, T]
$$

In addition, if

$$
\widetilde{\mathbf{u}} \in \mathcal{C}\left(0, T ; H^{3}(\Omega)^{d}\right), \widetilde{\theta} \in \mathcal{C}\left(0, T ; H^{2}(\Omega)\right) \quad \text { and } \quad \operatorname{tr} \mathbf{B}^{-1} \in \mathcal{C}\left(0, T ; H^{2}(\Omega)\right),
$$


then $\mathbf{B}_{t} \in L^{\infty}\left(0, T ; H^{1}(\Omega)^{d \times d}\right)$ and

$$
\begin{aligned}
\left\|\mathbf{B}_{t}(t)\right\|_{1} \leq & C\|\widetilde{\theta}(t)\|_{2}\left[\left(\left\|\mathbf{B}_{0}\right\|_{2}^{2}+\left\|\mathbf{B}_{0}\right\|_{2}^{4}\right)\left\|\mathbf{B}_{0}\right\|_{2}\right]^{1 / 2}\left[\left(\left\|\mathbf{B}_{0}\right\|_{2}^{3}+\left\|\mathbf{B}_{0}\right\|_{2}^{6}\right)\left\|\mathbf{B}_{0}\right\|_{2}^{2}\right]^{1 / 2} \\
& +C\left(\|\widetilde{\mathbf{u}}(t)\|_{3}+\|\widetilde{\theta}(t)\|_{2}\right)\left\|\mathbf{B}_{0}\right\|_{2}+C\|\widetilde{\theta}(t)\|_{2}\left[\left\|\mathbf{B}_{0}\right\|_{2}\right. \\
& \left.+\left\|\mathbf{B}_{0}\right\|_{2}^{2}\right]^{1 / 2}\left[\left(\left\|\mathbf{B}_{0}\right\|_{2}^{2}+\left\|\mathbf{B}_{0}\right\|_{2}^{4}\right)\left\|\mathbf{B}_{0}\right\|_{2}\right]^{1 / 2}
\end{aligned}
$$

Proof. We just need to derive the bounds (4.18) and (4.19). For that purpose we recall some well-known inequalities that will be used frequently.

First, Young's inequality states that

$$
a b \leq \frac{\epsilon}{p} a^{p}+\frac{1}{q \epsilon^{q / p}} b^{q} \quad \text { for all } a, b, \epsilon>0, \quad \text { with } \frac{1}{p}+\frac{1}{q}=1 .
$$

The following interpolation inequality ${ }^{3}$ (p. 195) will also be used: for $1 \leq p \leq q<\infty$ there exists a positive constant $C \equiv C(p, q, \Omega)$, such that

$$
\|u\|_{L^{q}(\Omega)} \leq C\|u\|_{L^{p}(\Omega)}^{1-\alpha}\|u\|_{W^{1, d}(\Omega)}^{\alpha} \quad \text { with } \alpha=1-\frac{p}{q} .
$$

The following Sobolev inequality ${ }^{6}$ will be employed for $\Omega \subset \mathbb{R}^{3}$ : there exists a constant $C \equiv C(\Omega)$, such that for all $v \in H^{1}(\Omega)$,

$$
\begin{aligned}
\|v\|_{L^{3}(\Omega)} & \leq C\|v\|^{1 / 2}\|v\|_{1}^{1 / 2}, \\
\|v\|_{L^{6}(\Omega)} & \leq C\|v\|_{1} .
\end{aligned}
$$

We will also use the following ${ }^{31}$ (p. 53):

Multiplicative algebra. If $\Omega \subset \mathbb{R}^{d}$ is of class $C^{m}$, and $m>d / 2$, then $H^{m}(\Omega)$ is a multiplicative algebra. That is, one can find $C \equiv C(\Omega)$ such that if $\mathbf{u}, \mathbf{v} \in H^{m}(\Omega)$, then $\mathbf{u} \cdot \mathbf{v} \in H^{m}(\Omega)$ and

$$
\|\mathbf{u} \cdot \mathbf{v}\|_{m} \leq C\|\mathbf{u}\|_{m}\|\mathbf{v}\|_{m} .
$$

We also recall that for $\mathbf{v} \in V$ and $\mathbf{B}$ sufficiently smooth, one has

$$
\left([\mathbf{v} \cdot \nabla] \partial^{\beta} \mathbf{B}, \partial^{\beta} \mathbf{B}\right)=0 .
$$

Estimates for the solution of (4.13). A standard energy estimate for Eq. (4.13) gives (see Refs. 7 and 23)

$$
\begin{aligned}
\frac{1}{2} \frac{d}{d t}\|\mathbf{B}(t)\|_{2}^{2}= & -\sum_{|\beta| \leq 2}\left(\partial^{\beta}([\widetilde{\mathbf{u}} \cdot \nabla] \mathbf{B}), \partial^{\beta} \mathbf{B}\right) \\
& +\sum_{|\beta| \leq 2}\left(\partial^{\beta}\left([\nabla \widetilde{\mathbf{u}}] \mathbf{B}+\mathbf{B}[\nabla \widetilde{\mathbf{u}}]^{\mathrm{T}}\right), \partial^{\beta} \mathbf{B}\right) \\
& +\alpha_{2} \sum_{|\beta| \leq 2}\left(\partial^{\beta}\left[\widetilde{\theta}\left(\frac{d}{\operatorname{tr} \mathbf{B}^{-1}} \mathbf{I}-\mathbf{B}\right)\right], \partial^{\beta} \mathbf{B}\right) .
\end{aligned}
$$

We now estimate the terms on the right-hand side of (4.25). 
Starting with the first term on the right-hand side, from (4.24) and (4.2) we have

$$
\begin{aligned}
& \sum_{|\beta| \leq 2}\left(\partial^{\beta}([\widetilde{\mathbf{u}} \cdot \nabla] \mathbf{B}), \partial^{\beta} \mathbf{B}\right) \\
&=([\widetilde{\mathbf{u}} \cdot \nabla] \mathbf{B}, \mathbf{B}) \\
&+\sum_{|\beta|=1}\left(\partial^{\beta}([\widetilde{\mathbf{u}} \cdot \nabla] \mathbf{B}), \partial^{\beta} \mathbf{B}\right)+\sum_{|\beta|=2}\left(\partial^{\beta}([\widetilde{\mathbf{u}} \cdot \nabla] \mathbf{B}), \partial^{\beta} \mathbf{B}\right) \\
&=0+\sum_{|\beta|=1}\left(\left[\partial^{\beta} \widetilde{\mathbf{u}} \cdot \nabla\right] \mathbf{B}+[\widetilde{\mathbf{u}} \cdot \nabla] \partial^{\beta} \mathbf{B}, \partial^{\beta} \mathbf{B}\right) \\
& \\
&+\sum_{|\beta|=2}\left(\left[\partial^{\beta} \widetilde{\mathbf{u}} \cdot \nabla\right] \mathbf{B}+\sum_{|\alpha|=1}\left(\begin{array}{c}
\alpha \\
\beta
\end{array}\right)\left[\partial^{\alpha} \widetilde{\mathbf{u}} \cdot \nabla\right] \partial^{\beta-\alpha} \mathbf{B}+[\widetilde{\mathbf{u}} \cdot \nabla] \partial^{\beta} \mathbf{B}, \partial^{\beta} \mathbf{B}\right) \\
& \leq C\left(\|\nabla \widetilde{\mathbf{u}}\|_{L^{\infty}}\|\mathbf{B}\|_{2}^{2}+\left\|\left[\partial^{2} \widetilde{\mathbf{u}} \cdot \nabla\right] \mathbf{B}\right\|\|\mathbf{B}\|_{2}\right) .
\end{aligned}
$$

When $d=2$, thanks to Hölder's inequality and (4.21),

$$
\begin{aligned}
\left\|\left[\partial^{2} \widetilde{\mathbf{u}} \cdot \nabla\right] \mathbf{B}\right\| & =\left\|\partial^{2} \widetilde{\mathbf{u}}\right\|_{L^{4}}\|\nabla \mathbf{B}\|_{L^{4}} \\
& \leq C\left\|\partial^{2} \widetilde{\mathbf{u}}\right\|^{1 / 2}\left\|\partial^{2} \widetilde{\mathbf{u}}\right\|_{1}^{1 / 2}\|\nabla \mathbf{B}\|^{1 / 2}\|\nabla \mathbf{B}\|_{1}^{1 / 2} \\
& \leq C\|\widetilde{\mathbf{u}}\|_{3}\|\mathbf{B}\|_{2},
\end{aligned}
$$

and when $d=3$, using Hölder's inequality and (4.22),

$$
\begin{aligned}
\left\|\partial^{2} \widetilde{\mathbf{u}} \cdot \nabla \mathbf{B}\right\| & \leq\left\|\partial^{2} \widetilde{\mathbf{u}}\right\|_{L^{6}}\|\nabla \mathbf{B}\|_{L^{3}} \\
& \leq C\left\|\partial^{2} \widetilde{\mathbf{u}}\right\|_{1}\|\nabla \mathbf{B}\|_{1} \\
& \leq C\|\widetilde{\mathbf{u}}\|_{3}\|\mathbf{B}\|_{2} .
\end{aligned}
$$

Thus from (4.16), (4.27) and (4.26), we deduce that

$$
\sum_{|\beta| \leq 2}\left(\partial^{\beta}((\widetilde{\mathbf{u}} \cdot \nabla) \mathbf{B}), \partial^{\beta} \mathbf{B}\right) \leq C\|\widetilde{\mathbf{u}}\|_{3}\|\mathbf{B}\|_{2}^{2} .
$$

Next, the process in getting (4.28) and use of (4.23), yield

$$
\begin{aligned}
\sum_{|\beta| \leq 2}\left(\partial^{\beta}\left([\nabla \widetilde{\mathbf{u}}] \mathbf{B}+\mathbf{B}[\nabla \widetilde{\mathbf{u}}]^{\mathrm{T}}\right), \partial^{\beta} \mathbf{B}\right) & \leq 2 \sum_{|\beta| \leq 2}\left\|\partial^{\beta}(\nabla \widetilde{\mathbf{u}} \mathbf{B})\right\|\left\|\partial^{\beta} \mathbf{B}\right\| \\
& \leq C\|\nabla \widetilde{\mathbf{u}} \mathbf{B}\|_{2}\|\mathbf{B}\|_{2} \\
& \leq C\|\widetilde{\mathbf{u}}\|_{3}\|\mathbf{B}\|_{2}^{2} .
\end{aligned}
$$


Finally,

$$
\begin{aligned}
\sum_{|\beta| \leq 2} & \left(\partial^{\beta} \widetilde{\theta}\left(\frac{d}{\operatorname{tr} \mathbf{B}^{-1}} \mathbf{I}-\mathbf{B}\right), \partial^{\beta} \mathbf{B}\right)=\left(\widetilde{\theta}\left(\frac{d}{\operatorname{tr} \mathbf{B}^{-1}} \mathbf{I}-\mathbf{B}\right), \mathbf{B}\right) \\
+ & \left(\partial \widetilde{\theta}\left(\frac{d}{\operatorname{tr} \mathbf{B}^{-1}} \mathbf{I}-\mathbf{B}\right), \partial \mathbf{B}\right)+\left(\widetilde{\theta}\left(\partial \frac{d}{\operatorname{tr} \mathbf{B}^{-1}} \mathbf{I}-\partial \mathbf{B}\right), \partial \mathbf{B}\right) \\
& +\left(\partial^{2} \widetilde{\theta}\left(\frac{d}{\operatorname{tr} \mathbf{B}^{-1}} \mathbf{I}-\mathbf{B}\right), \partial^{2} \mathbf{B}\right)+\left(\widetilde{\theta}\left(\partial^{2} \frac{d}{\operatorname{tr} \mathbf{B}^{-1}} \mathbf{I}-\partial^{2} \mathbf{B}\right), \partial^{2} \mathbf{B}\right) .
\end{aligned}
$$

We now estimate each term on the right-hand side of (4.30). For that purpose we first recall that if $\mathbf{E}$ is symmetric and positive-definite matrix of size $d$, then

$$
E_{i j}^{2} \leq E_{i i} E_{j j} \quad \text { for all } i \neq j,
$$

and

$$
[\operatorname{det} \mathbf{E}]^{1 / d} \leq(1 / d) \operatorname{tr} \mathbf{E} .
$$

Now, observing that $\mathbf{B}^{-1}$ is symmetric, positive-definite, from $(4.31)_{2}$, and the fact that $\operatorname{det} \mathbf{B}^{-1}=1$ (see Lemma 4.3), the inequality tr $\mathbf{B}^{-1} \geq d$ holds. Combining this with (4.16), it follows that

$$
\begin{gathered}
\left(\widetilde{\theta}\left(\frac{d}{\operatorname{tr} \mathbf{B}^{-1}} \mathbf{I}-\mathbf{B}\right), \mathbf{B}\right) \leq C\|\widetilde{\theta}\|\|\mathbf{B}\|+C\|\widetilde{\theta}\|_{2}\|\mathbf{B}\|_{2}^{2}, \\
\left(\partial \widetilde{\theta}\left(\frac{d}{\operatorname{tr} \mathbf{B}^{-1}} \mathbf{I}-\mathbf{B}\right), \partial \mathbf{B}\right) \leq C\|\widetilde{\theta}\|_{1}\|\mathbf{B}\|_{1}+C\|\widetilde{\theta}\|_{2}\|\mathbf{B}\|_{2}^{2}, \\
\left(\partial^{2} \widetilde{\theta}\left(\frac{d}{\operatorname{tr} \mathbf{B}^{-1}} \mathbf{I}-\mathbf{B}\right), \partial^{2} \mathbf{B}\right) \leq C\|\widetilde{\theta}\|_{2}\|\mathbf{B}\|_{2}+C\|\widetilde{\theta}\|_{2}\|\mathbf{B}\|_{2}^{2} .
\end{gathered}
$$

Next, we let $\mathcal{H}(\mathbf{B})=1 / \operatorname{tr} \mathbf{B}^{-1}$. From Gateaux-differentiability, and the identity (4.15), one gets

$$
\begin{aligned}
\partial \mathcal{H}(\mathbf{B}) \cdot \mathbf{A}= & \frac{\operatorname{tr}\left[\mathbf{B}^{-1} \mathbf{A} \mathbf{B}^{-1}\right]}{\left[\operatorname{tr} \mathbf{B}^{-1}\right]^{2}}, \\
\partial^{2} \mathcal{H}(\mathbf{B}) \cdot(\mathbf{A}, \mathbf{C})= & \frac{2 \operatorname{tr}\left[\mathbf{B}^{-1} \mathbf{A} \mathbf{B}^{-1}\right] \operatorname{tr}\left[\mathbf{B}^{-1} \mathbf{C B}^{-1}\right]}{\left[\operatorname{tr} \mathbf{B}^{-1}\right]^{3}} \\
& -\frac{\operatorname{tr}\left[\mathbf{B}^{-1} \mathbf{A} \mathbf{B}^{-1} \mathbf{C} \mathbf{B}^{-1}\right]+\operatorname{tr}\left[\mathbf{B}^{-1} \mathbf{C B}^{-1} \mathbf{A} \mathbf{B}^{-1}\right]}{\left[\operatorname{tr} \mathbf{B}^{-1}\right]^{2}} .
\end{aligned}
$$

One readily observes that
(a) $\partial^{2} \mathcal{H}(\mathbf{B})(\cdot, \cdot)$ is symmetric,
(b) $\partial \mathcal{H}(\mathbf{B})=\partial \mathcal{H}(\mathbf{B}) \cdot \partial \mathbf{B}=\frac{\operatorname{tr}\left[\mathbf{B}^{-1} \partial \mathbf{B} \mathbf{B}^{-1}\right]}{\left[\operatorname{tr} \mathbf{B}^{-1}\right]^{2}}$, 
(c)

$$
\begin{aligned}
\partial^{2} \mathcal{H}(\mathbf{B}) & =\partial^{2} \mathcal{H}(\mathbf{B}) \cdot(\partial \mathbf{B}, \partial \mathbf{B}) \\
& =\frac{2\left[\operatorname{tr}\left[\mathbf{B}^{-1} \partial \mathbf{B} \mathbf{B}^{-1}\right]\right]^{2}}{\left[\operatorname{tr} \mathbf{B}^{-1}\right]^{3}}-\frac{2 \operatorname{tr}\left[\mathbf{B}^{-1} \partial \mathbf{B} \mathbf{B}^{-1} \partial \mathbf{B} \mathbf{B}^{-1}\right]}{\left[\operatorname{tr} \mathbf{B}^{-1}\right]^{2}} .
\end{aligned}
$$

Now, working in a specific direction, say $x$, and using the fact that $\mathbf{B}^{-1}$ is symmetric, positive semi-definite, and $\operatorname{det} \mathbf{B}^{-1}=1$, from $(4.31)_{1}$, one obtains

$$
\begin{aligned}
\partial_{x} \mathcal{H}(\mathbf{B}) & =\frac{B_{i j}^{-1}\left[\partial_{x} B_{j k}\right] B_{k i}^{-1}}{\left[B_{i i}^{-1}\right]^{2}} \leq \frac{\left|\left[\partial_{x} B_{j k}\right]\right|\left[B_{j j}^{-1} B_{k k}^{-1}\right]^{1 / 2}}{B_{i i}^{-1}} \leq\left|\partial_{x} \mathbf{B}\right|, \\
\partial_{x x}^{2} \mathcal{H}(\mathbf{B}) & =\frac{2\left[B_{i j}^{-1}\right]^{2}\left[\partial_{x} B_{j k}\right]^{2}\left[B_{k i}^{-1}\right]^{2}}{\left[B_{i i}^{-1}\right]^{3}}-\frac{2\left[B_{i j}^{-1}\right]\left[\partial_{x} B_{j k}\right]\left[B_{k l}^{-1}\right]\left[\partial_{x} B_{l m}\right]\left[B_{m i}^{-1}\right]}{\left[B_{i i}^{-1}\right]^{2}} \\
& \leq 2\left|\partial_{x} \mathbf{B}\right|^{2}\left[\operatorname{tr} \mathbf{B}^{-1}\right]+2\left|\partial_{x} \mathbf{B}\right|^{2}\left|\mathbf{B}^{-1}\right| .
\end{aligned}
$$

Next, repeating the above computations in each direction, we find that

$$
\left\{\begin{array}{l}
|\partial \mathcal{H}(\mathbf{B})| \leq C|\partial \mathbf{B}| \\
\left|\partial^{2} \mathcal{H}(\mathbf{B})\right| \leq C|\partial \mathbf{B}|^{2}\left[\operatorname{tr} \mathbf{B}^{-1}\right]+C|\partial \mathbf{B}|^{2}\left|\mathbf{B}^{-1}\right| \leq C|\partial \mathbf{B}|^{2}\left|\mathbf{B}^{-1}\right|
\end{array}\right.
$$

Hence,

$$
\begin{aligned}
\left(\widetilde{\theta}\left(\partial \frac{d}{\operatorname{tr} \mathbf{B}^{-1}} \mathbf{I}-\partial \mathbf{B}\right), \partial \mathbf{B}\right) & \leq C(|\partial \mathbf{B}|, \widetilde{\theta}|\partial \mathbf{B}|) \leq C\|\widetilde{\theta}\|_{1}\|\mathbf{B}\|_{1}^{2} \\
\left(\widetilde{\theta}\left(\partial^{2} \frac{d}{\operatorname{tr} \mathbf{B}^{-1}} \mathbf{I}-\partial^{2} \mathbf{B}\right), \partial^{2} \mathbf{B}\right) & \leq C\left(\left|\mathbf{B}^{-1} \| \partial \mathbf{B}\right|^{2}\left|\partial^{2} \mathbf{B}\right|, \widetilde{\theta}\right)+\left(\widetilde{\theta}\left|\partial^{2} \mathbf{B}\right|,\left|\partial^{2} \mathbf{B}\right|\right) \\
& \leq C\|\widetilde{\theta}\|_{2}\|\mathbf{B}\|_{2}^{3}\left\|\mathbf{B}^{-1}\right\|_{2}+C\|\widetilde{\theta}\|_{2}\|\mathbf{B}\|_{2}^{2} .
\end{aligned}
$$

It is apparent that to close the estimates in $(4.35)_{2}$, we need to estimate $\left\|\mathbf{B}^{-1}\right\|_{2}$. For that purpose, since $\operatorname{det} \mathbf{B}=1$, in two space dimensions we have

$$
\left\|\mathbf{B}^{-1}\right\|_{2}=\|\operatorname{cof} \mathbf{B}\|_{2}=\|\mathbf{B}\|_{2},
$$

while in three space dimensions,

$$
\left\|\mathbf{B}^{-1}\right\|_{2}=\|\operatorname{cof} \mathbf{B}\|_{2} \leq C\|\mathbf{B}\|_{2}^{2}
$$

Therefore,

$$
\left\|\mathbf{B}^{-1}\right\|_{2} \leq C\left(\|\mathbf{B}\|_{2}+\|\mathbf{B}\|_{2}^{2}\right)
$$

Thus

$$
\begin{aligned}
\left(\tilde{\theta}\left(\partial \frac{d}{\operatorname{tr} \mathbf{B}^{-1}} \mathbf{I}-\partial \mathbf{B}\right), \partial \mathbf{B}\right) & \leq C\|\widetilde{\theta}\|_{1}\|\mathbf{B}\|_{1}^{2}, \\
\left(\tilde{\theta}\left(\partial^{2} \frac{d}{\operatorname{tr} \mathbf{B}^{-1}} \mathbf{I}-\partial^{2} \mathbf{B}\right), \partial^{2} \mathbf{B}\right) & \leq C\|\widetilde{\theta}\|_{2}\|\mathbf{B}\|_{2}^{3}\left\{\|\mathbf{B}\|_{2}+\|\mathbf{B}\|_{2}^{2}\right\}+C\|\widetilde{\theta}\|_{2}\|\mathbf{B}\|_{2}^{2} .
\end{aligned}
$$


Returning to (4.30) with (4.37), and (4.32), one obtains

$$
\begin{aligned}
& \sum_{|\beta| \leq 2}\left(\partial^{\beta} \widetilde{\theta}\left(\frac{d}{\operatorname{tr} \mathbf{B}^{-1}} \mathbf{I}-\mathbf{B}\right), \partial^{\beta} \mathbf{B}\right) \\
& \quad \leq C\|\widetilde{\theta}\|_{2}\|\mathbf{B}\|_{2}+C\|\widetilde{\theta}\|_{2}\|\mathbf{B}\|_{2}^{2}+C\|\widetilde{\theta}\|_{2}\|\mathbf{B}\|_{2}^{4}+C\|\widetilde{\theta}\|_{2}\|\mathbf{B}\|_{2}^{5} .
\end{aligned}
$$

Putting together (4.25) with (4.28), (4.29), (4.38), and (4.20) one has

$$
\begin{aligned}
\frac{d}{d t}\|\mathbf{B}(t)\|_{2}^{2} & \leq C\left(\|\widetilde{\mathbf{u}}\|_{3}+\|\widetilde{\theta}\|_{2}\right)\|\mathbf{B}\|_{2}^{2}+C\|\widetilde{\theta}\|_{2}\|\mathbf{B}\|_{2}+C\|\widetilde{\theta}\|_{2}\|\mathbf{B}\|_{2}^{4}+C\|\widetilde{\theta}\|_{2}\|\mathbf{B}\|_{2}^{5} \\
& \leq C\left(1+\|\widetilde{\mathbf{u}}\|_{3}+\|\widetilde{\theta}\|_{2}+\|\widetilde{\theta}\|_{2}^{2}\right)\left(1+\|\mathbf{B}\|_{2}^{2}\right)^{5},
\end{aligned}
$$

from which we deduce that

$$
\|\mathbf{B}(t)\|_{2}^{4} \leq \frac{\left\|\mathbf{B}_{0}\right\|_{2}^{4}}{1-C\left\|\mathbf{B}_{0}\right\|_{2}^{4}\left(\|\widetilde{\mathbf{u}}\|_{L^{1}\left(0, t ; H^{3}\right)}+\|\widetilde{\theta}\|_{L^{1}\left(0, t ; H^{2}\right)}+\|\widetilde{\theta}\|_{L^{2}\left(0, t ; H^{2}\right)}^{2}\right)},
$$

provided that (4.17) is satisfied. We select $T$ such that

$$
T\left[\|\widetilde{\mathbf{u}}\|_{L^{\infty}\left(0, T ; H^{3}\right)}+\|\widetilde{\theta}\|_{L^{\infty}\left(0, T ; H^{2}\right)}+\|\widetilde{\theta}\|_{L^{\infty}\left(0, T ; H^{2}\right)}^{2}\right] \leq \frac{1}{C\left\|\mathbf{B}_{0}\right\|_{2}^{4}} .
$$

Thus (4.40), and (4.41) gives the desired inequality (4.18).

In order to show that $\mathbf{B}_{t} \in L^{\infty}\left(0, T ; L^{2}(\Omega)^{d \times d}\right)$, we take the $L^{2}$-norm on both sides of $\mathbf{B}$-equation of (4.13), which gives

$$
\begin{aligned}
\left\|\mathbf{B}_{t}(t)\right\| \leq & \| \widetilde{\mathbf{u}}(t) \cdot \nabla] \mathbf{B}(t)\|+\|[\nabla \widetilde{\mathbf{u}}(t)] \mathbf{B}(t)\|+\| \mathbf{B}(t)[\nabla \widetilde{\mathbf{u}}(t)]^{\mathrm{T}} \| \\
& +\alpha_{2}\left\|\widetilde{\theta}(t)\left[\frac{d}{\operatorname{tr} \mathbf{B}^{-1}(t)} \mathbf{I}-\mathbf{B}(t)\right]\right\| \\
\leq & C\|\widetilde{\mathbf{u}}(t)\|_{2}\|\mathbf{B}(t)\|_{2}+C\left\|\frac{\widetilde{\theta}(t)}{\operatorname{tr} \mathbf{B}^{-1}(t)}\right\|+C\|\widetilde{\theta}(t) \mathbf{B}(t)\| \\
\leq & C\|\widetilde{\mathbf{u}}(t)\|_{2}\|\mathbf{B}(t)\|_{2}+C\|\widetilde{\theta}(t)\|+C\|\widetilde{\theta}(t)\|_{2}\|\mathbf{B}(t)\|_{2}<\infty,
\end{aligned}
$$

where we have used the inequality $\operatorname{tr} \mathbf{B}^{-1}>d,(4.18)$, and similar arguments as in the proofs of (4.27) and (4.26). This shows that $\mathbf{B}_{t} \in L^{2}\left(0, T ; L^{2}(\Omega)^{d \times d}\right)$.

Finally, if (4.13) is written in the form

$$
\mathbf{B}_{t}=\underbrace{-[\widetilde{\mathbf{u}} \cdot \nabla] \mathbf{B}+[\nabla \widetilde{\mathbf{u}}] \mathbf{B}+\mathbf{B}[\nabla \widetilde{\mathbf{u}}]^{\mathrm{T}}+\alpha_{2} \widetilde{\theta}\left[\frac{d}{\operatorname{tr} \mathbf{B}^{-1}} I-\mathbf{B}\right]}_{G(\widetilde{\mathbf{u}} \widetilde{\theta}, \mathbf{B})},
$$

then by integrating $(4.42)$ over $(0, t)$ one gets

$$
\mathbf{B}(t)=\mathbf{B}_{0}+\int_{0}^{t} G(\widetilde{\mathbf{u}}, \widetilde{\theta}, \mathbf{B}(s)) d s .
$$


It is manifest that if $\widetilde{\mathbf{u}} \in \mathcal{C}\left(0, T ; H^{3}(\Omega)^{d}\right), \widetilde{\theta} \in \mathcal{C}\left(0, T ; H^{2}(\Omega)\right)$, $\operatorname{tr} \mathbf{B}^{-1} \in$ $\mathcal{C}\left(0, T ; H^{2}(\Omega)\right)$, with $\mathbf{B} \in \mathcal{C}\left(0, T ; H^{2}(\Omega)^{d \times d}\right)$, then $G(\widetilde{\mathbf{u}}, \widetilde{\theta}, \mathbf{B}) \in \mathcal{C}\left(0, T ; H^{1}\right)$.

Hence taking the $H^{1}$-norm on both sides of (4.42), one gets

$$
\begin{aligned}
\left\|\partial_{t} \mathbf{B}(t)\right\|_{1} \leq & \|[\widetilde{\mathbf{u}}(t) \cdot \nabla] \mathbf{B}(t)\|_{1}+2\|\nabla \widetilde{\mathbf{u}}(t) \mathbf{B}(t)\|_{1}+\alpha_{2}\left\|\widetilde{\theta}(t)\left[\frac{d}{\operatorname{tr} \mathbf{B}^{-1}(t)} \mathbf{I}-\mathbf{B}(t)\right]\right\|_{1} \\
\leq & \|[\widetilde{\mathbf{u}}(t) \cdot \nabla] \mathbf{B}(t)\|_{1}+C\|\widetilde{\mathbf{u}}(t)\|_{3}\|\mathbf{B}(t)\|_{2} \\
& +C\left\|\frac{\widetilde{\theta}(t)}{\operatorname{tr} \mathbf{B}^{-1}(t)}\right\|_{1}+C\|\widetilde{\theta}(t) \mathbf{B}(t)\|_{1} .
\end{aligned}
$$

We now need to bound expressions on the right-hand side of (4.44).

First, since $\left.[\widetilde{\mathbf{u}}(t) \cdot \nabla] \mathbf{B}(t)\right|_{\partial \Omega}=0$, Poincaré's inequality ensures that

$$
\begin{aligned}
\|[\widetilde{\mathbf{u}}(t) \cdot \nabla] \mathbf{B}(t)\|_{1} & \leq C\|\nabla[\widetilde{\mathbf{u}}(t) \cdot \nabla] \mathbf{B}(t)\| \\
& \leq C\|\nabla \widetilde{\mathbf{u}}(t) \cdot \nabla \mathbf{B}(t)\|+C\left\|\widetilde{\mathbf{u}}(t) \cdot \partial^{2} \mathbf{B}(t)\right\| \\
& \leq C\|\widetilde{\mathbf{u}}(t)\|_{2}\|\mathbf{B}(t)\|_{2} \\
& \leq C\|\widetilde{\mathbf{u}}(t)\|_{2}\left\|\mathbf{B}_{0}\right\|_{2} .
\end{aligned}
$$

Secondly, since $\left.\widetilde{\theta}(t) \mathbf{B}(t)\right|_{\partial \Omega}=0$, similar arguments as in the proof of (4.45) yield

$$
\begin{aligned}
\|\widetilde{\theta}(t) \mathbf{B}(t)\|_{1} & \leq C\|\nabla(\widetilde{\theta}(t) \mathbf{B}(t))\| \\
& \leq C\|\nabla \widetilde{\theta}(t) \mathbf{B}(t)\|+C\|\widetilde{\theta}(t) \nabla \mathbf{B}(t)\| \\
& \leq C\|\widetilde{\theta}(t)\|_{2}\|\mathbf{B}(t)\|_{2} \\
& \leq C\|\widetilde{\theta}(t)\|_{2}\left\|\mathbf{B}_{0}\right\|_{2} .
\end{aligned}
$$

Thirdly $\left.\widetilde{\theta}(t)\left[\operatorname{tr} \mathbf{B}^{-1}(t)\right]^{-1}\right|_{\partial \Omega}=0$, so that

$$
\left\|\frac{\widetilde{\theta}(t)}{\operatorname{tr} \mathbf{B}^{-1}(t)}\right\|_{1} \leq C\left\|\partial \frac{\widetilde{\theta}(t)}{\operatorname{tr} \mathbf{B}^{-1}(t)}\right\| \leq C\left\|\frac{\nabla \widetilde{\theta}(t) \operatorname{tr} \mathbf{B}^{-1}(t)-\widetilde{\theta}(t) \partial \operatorname{tr} \mathbf{B}^{-1}(t)}{\operatorname{tr}^{2} \mathbf{B}^{-1}(t)}\right\| .
$$

But since $\mathbf{B}^{-1}$ is symmetric and positive definite, (4.31) applies and one has

$$
\frac{1}{\operatorname{tr} \mathbf{B}^{-1}(t)} \leq \frac{1}{d\left[\operatorname{det} \mathbf{B}^{-1}(t)\right]^{1 / d}} \leq \frac{1}{d} .
$$

Hence

$$
\begin{aligned}
\left\|\frac{\widetilde{\theta}(t)}{\operatorname{tr} \mathbf{B}^{-1}(t)}\right\|_{1} & \leq \frac{C}{d^{2}}\left\|\nabla \widetilde{\theta}(t) \operatorname{tr} \mathbf{B}^{-1}(t)-\widetilde{\theta}(t) \partial \operatorname{tr} \mathbf{B}^{-1}(t)\right\| \\
& \leq C\left\|\nabla \widetilde{\theta}(t) \operatorname{tr} \mathbf{B}^{-1}(t)\right\|+C\left\|\widetilde{\theta}(t) \partial \operatorname{tr} \mathbf{B}^{-1}(t)\right\| .
\end{aligned}
$$


By similar arguments as in the proofs of (4.27) and (4.26), one has

$$
\begin{aligned}
\left\|\nabla \widetilde{\theta}(t) \operatorname{tr} \mathbf{B}^{-1}(t)\right\| & \leq C\|\widetilde{\theta}(t)\|_{2}\left\|\mathbf{B}^{-1}(t)\right\|^{1 / 2}\left\|\partial \operatorname{tr} \mathbf{B}^{-1}(t)\right\|^{1 / 2}, \\
\left\|\widetilde{\theta}(t) \partial \operatorname{tr} \mathbf{B}^{-1}(t)\right\| & \leq C\|\widetilde{\theta}(t)\|_{1}\left\|\partial \operatorname{tr} \mathbf{B}^{-1}(t)\right\|^{1 / 2}\left\|\partial^{2} \operatorname{tr} \mathbf{B}^{-1}(t)\right\|^{1 / 2} .
\end{aligned}
$$

In order to treat $\left\|\partial \operatorname{tr} \mathbf{B}^{-1}(t)\right\|$ and $\left\|\partial^{2} \operatorname{tr} \mathbf{B}^{-1}(t)\right\|$, we let $\mathcal{K}(\mathbf{B})=\operatorname{tr} \mathbf{B}^{-1}$. From Gateaux-differentiability, and the identity (4.15),

$$
\partial \mathcal{K}(\mathbf{B}) \cdot \mathbf{A}=-\operatorname{tr}\left[\mathbf{B}^{-1} \mathbf{A} \mathbf{B}^{-1}\right]
$$

$\partial^{2} \mathcal{K}(\mathbf{B}) \cdot(\mathbf{A}, \mathbf{C})=\partial(\partial \mathcal{K}(\mathbf{B}) \cdot \mathbf{A}) \cdot \mathbf{C}=\operatorname{tr}\left[\mathbf{B}^{-1} \mathbf{C B}^{-1} \mathbf{A} \mathbf{B}^{-1}\right]+\operatorname{tr}\left[\mathbf{B}^{-1} \mathbf{A B}^{-1} \mathbf{C B}^{-1}\right]$.

Thus

$$
\begin{aligned}
\partial \operatorname{tr} \mathbf{B}^{-1} & =\partial \mathcal{K}(\mathbf{B}) \cdot \partial \mathbf{B}=-\operatorname{tr}\left(\mathbf{B}^{-1} \partial \mathbf{B} \mathbf{B}^{-1}\right), \\
\partial^{2} \operatorname{tr} \mathbf{B}^{-1} & =\partial^{2} \mathcal{K}(\mathbf{B}) \cdot(\partial \mathbf{B}, \partial \mathbf{B})=2 \operatorname{tr}\left(\mathbf{B}^{-1} \partial \mathbf{B} \mathbf{B}^{-1} \partial \mathbf{B} \mathbf{B}^{-1}\right) .
\end{aligned}
$$

By similar arguments as in the proofs of (4.27) and (4.26), one has

$$
\begin{aligned}
\left\|\partial \operatorname{tr} \mathbf{B}^{-1}(t)\right\| & \leq\left\|\operatorname{tr}\left(\mathbf{B}^{-1}(t) \partial \mathbf{B}^{-1}(t)\right)\right\| \leq C\left\|\left(\mathbf{B}^{-1}(t)\right)^{2} \partial \mathbf{B}(t)\right\| \\
& \leq C\left\|\mathbf{B}^{-1}(t)\right\|_{2}^{2}\|\mathbf{B}(t)\|_{2} \\
\left\|\partial^{2} \operatorname{tr} \mathbf{B}^{-1}(t)\right\| & \leq C\left\|\left(\mathbf{B}^{-1}(t)\right)^{3}(\partial \mathbf{B}(t))^{2}\right\| \leq C\left\|\mathbf{B}^{-1}(t)\right\|_{2}^{3}\|\mathbf{B}(t)\|_{2}^{2},
\end{aligned}
$$

which combined with (4.36) gives

$$
\begin{aligned}
\left\|\partial \operatorname{tr} \mathbf{B}^{-1}(t)\right\| & \leq C\left(\|\mathbf{B}(t)\|_{2}+\|\mathbf{B}(t)\|_{2}^{2}\right)^{2}\|\mathbf{B}(t)\|_{2} \leq C\left(\left\|\mathbf{B}_{0}\right\|_{2}^{2}+\left\|\mathbf{B}_{0}\right\|_{2}^{4}\right)\left\|\mathbf{B}_{0}\right\|_{2}, \\
\left\|\partial^{2} \operatorname{tr} \mathbf{B}^{-1}(t)\right\| & \leq C\left(\|\mathbf{B}(t)\|_{2}+\|\mathbf{B}(t)\|_{2}^{2}\right)^{3}\|\mathbf{B}(t)\|_{2}^{2} \leq C\left(\left\|\mathbf{B}_{0}\right\|_{2}^{3}+\left\|\mathbf{B}_{0}\right\|_{2}^{6}\right)\left\|\mathbf{B}_{0}\right\|_{2}^{2}
\end{aligned}
$$

Coming back to (4.48), we find that

$$
\begin{aligned}
\left\|\nabla \widetilde{\theta}(t) \operatorname{tr} \mathbf{B}^{-1}(t)\right\| \leq & C\|\widetilde{\theta}(t)\|_{2}\left[\left\|\mathbf{B}_{0}\right\|_{2}+\left\|\mathbf{B}_{0}\right\|_{2}^{2}\right]^{1 / 2}\left[\left(\left\|\mathbf{B}_{0}\right\|_{2}^{2}+\left\|\mathbf{B}_{0}\right\|_{2}^{4}\right)\left\|\mathbf{B}_{0}\right\|_{2}\right]^{1 / 2}, \\
\left\|\widetilde{\theta}(t) \partial \operatorname{tr} \mathbf{B}^{-1}(t)\right\| \leq & C\|\widetilde{\theta}(t)\|_{1}\left[\left(\left\|\mathbf{B}_{0}\right\|_{2}^{2}+\left\|\mathbf{B}_{0}\right\|_{2}^{4}\right)\left\|\mathbf{B}_{0}\right\|_{2}\right]^{1 / 2} \\
& \cdot\left[\left(\left\|\mathbf{B}_{0}\right\|_{2}^{3}+\left\|\mathbf{B}_{0}\right\|_{2}^{6}\right)\left\|\mathbf{B}_{0}\right\|_{2}^{2}\right]^{1 / 2} .
\end{aligned}
$$

Putting together (4.50) and (4.47), it follows that

$$
\begin{aligned}
\left\|\frac{\tilde{\theta}(t)}{\operatorname{tr} \mathbf{B}^{-1}(t)}\right\|_{1} \leq & C\|\tilde{\theta}(t)\|_{2}\left[\left\|\mathbf{B}_{0}\right\|_{2}+\left\|\mathbf{B}_{0}\right\|_{2}^{2}\right]^{1 / 2}\left[\left(\left\|\mathbf{B}_{0}\right\|_{2}^{2}+\left\|\mathbf{B}_{0}\right\|_{2}^{4}\right)\left\|\mathbf{B}_{0}\right\|_{2}\right]^{1 / 2} \\
& +C\|\widetilde{\theta}(t)\|_{2}\left[\left(\left\|\mathbf{B}_{0}\right\|_{2}^{2}+\left\|\mathbf{B}_{0}\right\|_{2}^{4}\right)\left\|\mathbf{B}_{0}\right\|_{2}\right]^{1 / 2}\left[\left(\left\|\mathbf{B}_{0}\right\|_{2}^{3}+\left\|\mathbf{B}_{0}\right\|_{2}^{6}\right)\left\|\mathbf{B}_{0}\right\|_{2}^{2}\right]^{1 / 2} .
\end{aligned}
$$

Returning to (4.44) with (4.45), (4.46) and (4.51), one finds the announced bound (4.19). 
The uniqueness of the solution $\mathbf{B}$ of (4.13) is obtained by using the energy estimate for the difference between two solutions. A similar approach is presented in the next section, so the proof is omitted here.

\section{Local Existence and Uniqueness of a Regular Solution}

In this section a fixed point strategy is used to construct regular solutions of (3.3). This approach was introduced by Guillopé and Saut ${ }^{9}$ in the context of the problem for Oldroyd B-fluids, and has also been used, inter alia, in Refs. 7 and 23 which are concerned with fiber suspension flows. A regular solution of (3.3) is understood to be any solution which satisfies the initial and boundary conditions in the usual sense, and which is smooth in the interior of $\Omega \times[0, T]$. As mentioned before, the question of constructing weak solutions in any suitable sense (i.e. Ref. 20 or 19) is open and is a nontrivial problem due to the complicated nature of the transport equation which does not have any dissipative mechanism, and the nonlinearities of the system.

The following is one of the main results of this section.

Theorem 5.1. Let $\Omega$ be a bounded open set of $\mathbb{R}^{d}$ and assume that $\Gamma$ is of class $C^{3}$. Assume further that $\mathbf{u}_{0} \in D(\mathbb{A}), \mathbf{B}_{0} \in X \cap H^{2}(\Omega)^{d \times d}$, with $\operatorname{det} \mathbf{B}_{0}=1, \theta_{0} \in$ $H^{2}(\Omega) \cap H_{0}^{1}(\Omega)$, and that $\mathbf{b}$ and $r$ satisfy the conditions

(i) $\mathbf{b}_{t} \in L^{2}\left(0, T ; H^{-1}(\Omega)^{d}\right), \mathbf{b} \in L^{2}\left(0, T ; H^{1}(\Omega)^{d}\right), \mathbf{b}(\mathbf{0}) \in L^{2}(\Omega)^{d}$,

(ii) $r \in H^{1}\left(0, T ; L^{2}(\Omega)\right)$.

Then there exists a positive constant $T_{0}$ such that (2.9) has only one solution $(\mathbf{B}, \mathbf{u}, p, \theta)$, with

(a) $\mathbf{B} \in \mathcal{C}\left(\left[0, T_{0}\right] ; X \cap H^{2}(\Omega)^{d \times d}\right)$,

(b) $\mathbf{u} \in \mathcal{C}\left(\left[0, T_{0}\right] ; D(\mathbb{A})\right) \cap L^{2}\left(\left(0, T_{0}\right) ; H^{3}(\Omega)\right), \mathbf{u}_{t} \in \mathcal{C}\left(\left[0, T_{0}\right] ; H\right) \cap L^{2}\left(\left(0, T_{0}\right) ; V\right)$,

(c) $\nabla p \in L^{2}\left(\left(0, T_{0}\right) ; H^{1}(\Omega)\right)$, and

(d) $\theta \in \mathcal{C}\left(\left[0, T_{0}\right] ; H^{2}(\Omega) \cap H_{0}^{1}(\Omega)\right)$, with $\theta_{t} \in \mathcal{C}\left(\left[0, T_{0}\right] ; L^{2}(\Omega)\right) \cap L^{2}\left(\left(0, T_{0}\right) ; H_{0}^{1}(\Omega)\right)$.

Proof. The proof proceeds in the following steps.

Step 1. Using the projection operator $\mathcal{P}$ introduced earlier, the $\mathbf{u}$-equation of (3.3) is equivalent to

$$
\mathbf{u}_{t}+\mathbb{A} \mathbf{u}=\mathbf{F}
$$

where

$$
\mathbf{F}(\mathbf{u}, \theta, \mathbf{B})=C_{1}(\mathbf{B} \nabla \theta+\theta \operatorname{div} \mathbf{B})-\mathcal{P}[\mathbf{u} \cdot \nabla] \mathbf{u}+\mathbf{b} .
$$

Now, for $(\widetilde{\mathbf{u}}, \widetilde{\theta}, \widetilde{\mathbf{B}})$ fixed, consider the problem of seeking $\mathbf{u}(t) \in D(\mathbb{A})$ such that

$$
\begin{aligned}
\mathbf{u}_{t}+\mathbb{A} \mathbf{u} & =\mathbf{F}(\widetilde{\mathbf{u}}, \widetilde{\theta}, \widetilde{\mathbf{B}}), \quad \text { a.e. for } t \in(0, T), \\
\mathbf{u}(0) & =\mathbf{u}_{0} .
\end{aligned}
$$


Step 2. Seek $\theta(t) \in H^{2}(\Omega) \cap H_{0}^{1}(\Omega)$ such that

$$
\begin{aligned}
\theta_{t}-\kappa \Delta \theta & =f(\widetilde{\mathbf{u}}, \widetilde{\theta}, \widetilde{\mathbf{B}}), \quad \text { a.e. in }(0, T), \\
\theta(0) & =\theta_{0},
\end{aligned}
$$

where $f$ is given by

$$
f(\mathbf{u}, \theta, \mathbf{B})=-[\mathbf{u} \cdot \nabla] \theta+\left[C_{1} \theta \mathbf{B}+2 \nu \mathbf{D}\right]: \mathbf{D}+r .
$$

From Lemmas 4.1 and 4.2, problems (5.1) and (5.2) are well-defined.

Next, for positive constants $T, K_{1}, K_{2}, K_{3}$ set $R(T)$

$$
=\left\{\begin{array}{l}
(\mathbf{v}, q, \mathbf{B}), \quad \text { with }(\mathbf{v}(\mathbf{0}), q(0), \mathbf{B}(\mathbf{0}))=\left(\mathbf{v}_{0}, q_{0}, \mathbf{B}_{0}\right), \\
\mathbf{v} \in L^{2}\left(0, T ; H^{3}(\Omega)^{d} \cap V\right) \cap \mathcal{C}(0, T ; D(\mathbb{A})), \mathbf{v}_{t} \in L^{2}(0, T ; V) \cap \mathcal{C}(0, T ; H), \\
q \in L^{\infty}\left(0, T ; H^{2}(\Omega)\right), q_{t} \in L^{\infty}\left(0, T ; L^{2}(\Omega)\right) \cap L^{2}\left(0, T ; H_{0}^{1}(\Omega)\right), \\
\mathbf{B} \in L^{\infty}\left(0, T ; H^{2}(\Omega)^{d \times d} \cap X\right), \mathbf{B}_{t} \in L^{\infty}\left(0, T ; H^{1}(\Omega)^{d \times d} \cap X\right), \\
\|\mathbf{v}\|_{L^{\infty}(0, T ; D(\mathbb{A})) \cap L^{2}\left(0, T ; H^{3}(\Omega)\right)}^{2}+\left\|\mathbf{v}_{t}\right\|_{L^{\infty}(0, T ; H) \cap L^{2}(0, T ; V)}^{2} \leq K_{1}, \\
\|q\|_{L^{\infty}\left(0, T ; H^{2}(\Omega)\right)}^{2}+\left\|q_{t}\right\|_{L^{\infty}\left(0, T ; L^{2}(\Omega)\right) \cap L^{2}\left(0, T ; H^{1}(\Omega)\right)}^{2} \leq K_{2}, \\
\|\mathbf{B}\|_{L^{\infty}\left(0, T ; H^{2}(\Omega)^{d \times d}\right)}^{2} \leq \max \left(K_{1}, K_{2}\right),\left\|\mathbf{B}_{t}\right\|_{L^{\infty}\left(0, T ; H^{1}(\Omega)^{d \times d}\right)}^{2} \leq K_{3} .
\end{array}\right\} .
$$

The set $R(T)$ has the following properties:

(i) $R(T)$ is non-empty. Indeed, let $(\mathbf{w}, \psi)$ be a solution of (4.5) and (4.8) with $(\mathbf{F}, f)=(\mathbf{0}, 0)$. Thus the estimates

$$
\begin{aligned}
& \|\mathbf{w}\|_{L^{2}\left(0, T ; H^{3}\right) \cap L^{\infty}(0, T ; D(\mathbb{A}))}^{2}+\left\|\mathbf{w}_{t}\right\|_{L^{2}(0, T ; V) \cap L^{\infty}(0, T ; H)}^{2} \leq C\left\|\mathbb{A} \mathbf{w}_{0}\right\|^{2}, \\
& \|\psi\|_{L^{\infty}\left(0, T ; H^{2}\right)}^{2}+\left\|\psi_{t}\right\|_{L^{\infty}\left(0, T ; L^{2}\right) \cap L^{2}\left(0, T ; H^{1}\right)}^{2} \leq C\left\|q_{0}\right\|_{2}^{2},
\end{aligned}
$$

are valid (see (4.7) and (4.12)). Let $K_{1}>C\left\|\mathbb{A}_{\mathbf{w}}\right\|^{2}+\left\|\mathbf{B}_{0}\right\|_{2}$ and $K_{2}>C\left\|\psi_{0}\right\|_{2}^{2}$. It is manifest that $\left(\mathbf{w}, \psi, \mathbf{B}_{0}\right) \in R(T)$.

(ii) $R(T)$ is a closed, convex subset of $\mathcal{C}\left(0, T ; H^{1}(\Omega)^{d}\right) \times \mathcal{C}\left(0, T ; H^{1}(\Omega)\right) \times$ $\mathcal{C}\left(0, T ; H^{1}(\Omega)^{d \times d}\right)$.

Step 3. We introduce the mapping

$\mathcal{L}: R(T) \rightarrow \mathcal{C}\left(0, T ; H^{1}(\Omega)^{d}\right) \times \mathcal{C}\left(0, T ; H^{1}(\Omega)\right) \times \mathcal{C}\left(0, T ; H^{1}(\Omega)^{d \times d}\right)$

$(\widetilde{\mathbf{u}}, \widetilde{\theta}, \widetilde{\mathbf{B}}) \rightarrow(\widehat{\mathbf{u}}, \widehat{\theta}, \widehat{\mathbf{B}}), \quad$ with $(\widehat{\mathbf{u}}, \widehat{\theta}, \widehat{\mathbf{B}})$ a solution of (5.1), (5.2) and (4.13).

The mapping $\mathcal{L}$ is well-defined, and a fixed point of $\mathcal{L}$, if it exists, will solve (3.3). From the Leray-Schauder theory ${ }^{6}$ it is therefore necessary to show that

(a) there exists a positive constant $T_{0}$, such that $\mathcal{L}\left(R\left(T_{0}\right)\right) \subset R\left(T_{0}\right)$; 
(b) the mapping $\mathcal{L}$ is compact;

(c) the mapping $\mathcal{L}$ is continuous.

For $(\widetilde{\mathbf{u}}, \widetilde{\theta}, \widetilde{\mathbf{B}}) \in R(T)$ we want $T_{0}>0$ such that $(\widehat{\mathbf{u}}, \widehat{\theta}, \widehat{\mathbf{B}})=\mathcal{L}(\widetilde{\mathbf{u}}, \widetilde{\theta}, \widetilde{\mathbf{B}}) \in R\left(T_{0}\right)$.

If $(\widehat{\mathbf{u}}, \widehat{\theta}, \widehat{\mathbf{B}})$ is the solution of (5.1), (5.2) and (4.13), then from (4.7),

$$
\begin{aligned}
& \|\widehat{\mathbf{u}}\|_{L^{2}\left(0, T ; H^{3}\right) \cap L^{\infty}(0, T ; D(\mathbb{A}))}^{2}+\left\|\widehat{\mathbf{u}}_{t}\right\|_{L^{2}(0, T ; V) \cap L^{\infty}(0, T ; H)}^{2} \\
& \quad \leq C\left[\left\|\mathbb{A} \mathbf{u}_{0}\right\|^{2}+\|\mathbf{F}\|_{L^{2}\left(0, T ; H^{1}\right)}^{2}+\left\|\mathbf{F}_{t}\right\|_{L^{2}\left(0, T ; H^{-1}\right)}^{2}+\|\mathbf{F}(\mathbf{0})\|^{2}\right],
\end{aligned}
$$

with

$$
\mathbf{F}=C_{1}(\widetilde{\mathbf{B}} \nabla \widetilde{\theta}+\widetilde{\theta} \operatorname{div} \widetilde{\mathbf{B}})-\mathcal{P}[\widetilde{\mathbf{u}} \cdot \nabla] \widetilde{\mathbf{u}}+\mathbf{b}
$$

It is therefore necessary to estimate $\|\mathbf{F}\|_{L^{2}\left(0, T ; H^{1}\right)}^{2}+\left\|\mathbf{F}_{t}\right\|_{L^{2}\left(0, T ; H^{-1}\right)}^{2}+\|\mathbf{F}(0)\|^{2}$.

Firstly,

$$
\begin{aligned}
\|\mathbf{F}(0)\| & \leq C_{1}(\|\widetilde{\mathbf{B}}(0) \nabla \widetilde{\theta}(0)\|+\|\widetilde{\theta}(0) \operatorname{div} \widetilde{\mathbf{B}}(0)\|)+\|\widetilde{\mathbf{u}}(0) \cdot \nabla \widetilde{\mathbf{u}}(0)\|+\|\mathbf{b}(0)\| \\
& =C_{1}\left(\left\|\mathbf{B}_{0} \nabla \theta_{0}\right\|+\left\|\theta_{0} \operatorname{div} \mathbf{B}_{0}\right\|\right)+\left\|\left(\mathbf{u}_{0} \cdot \nabla\right) \mathbf{u}_{0}\right\|+\|\mathbf{b}(\mathbf{0})\| \\
& \leq C\left\|\mathbf{B}_{0}\right\|_{2}\left\|\theta_{0}\right\|_{2}+C\left\|\mathbf{u}_{0}\right\|_{2}^{2}+\|\mathbf{b}(0)\| .
\end{aligned}
$$

Secondly, taking the $H^{1}$-norm on both sides of (5.5) and using (4.16) and (4.22), one gets

$$
\|\mathbf{F}\|_{1} \leq C\|\widetilde{\theta}\|_{2}\|\widetilde{\mathbf{B}}\|_{2}+C\|[\widetilde{\mathbf{u}} \cdot \nabla] \widetilde{\mathbf{u}}\|_{1}+\|\mathbf{b}\|_{1} .
$$

Now $\left.(\widetilde{\mathbf{u}} \cdot \nabla) \widetilde{\mathbf{u}}\right|_{\Gamma}=0$ so by Poincaré's inequality,

$$
\begin{aligned}
\|[\widetilde{\mathbf{u}} \cdot \nabla] \widetilde{\mathbf{u}}\|_{1} & \leq C\|\nabla([\widetilde{\mathbf{u}} \cdot \nabla] \widetilde{\mathbf{u}})\| \\
& =C\left\|(\nabla \widetilde{\mathbf{u}})^{2}+[\widetilde{\mathbf{u}} \cdot \nabla] \nabla \widetilde{\mathbf{u}}\right\| \\
& \leq C\left\|(\nabla \widetilde{\mathbf{u}})^{2}\right\|+C\|[\widetilde{\mathbf{u}} \cdot \nabla] \nabla \widetilde{\mathbf{u}}\| .
\end{aligned}
$$

In two-space dimensions, as in (4.26),

$$
\left\|(\nabla \widetilde{\mathbf{u}})^{2}\right\| \leq C\|\nabla \widetilde{\mathbf{u}}\|\left\|\nabla^{2} \widetilde{\mathbf{u}}\right\| \leq C\|\widetilde{\mathbf{u}}\|_{2}^{2},
$$

while in three-space dimensions, following (4.27),

$$
\left\|(\nabla \widetilde{\mathbf{u}})^{2}\right\| \leq C\|\nabla \widetilde{\mathbf{u}}\|^{1 / 2}\left\|\nabla^{2} \widetilde{\mathbf{u}}\right\|^{3 / 2} \leq C\|\widetilde{\mathbf{u}}\|_{2}^{2} .
$$

Similarly, one easily gets

$$
\|[\widetilde{\mathbf{u}} \cdot \nabla] \nabla \widetilde{\mathbf{u}}\| \leq C\|\nabla \widetilde{\mathbf{u}}\|\left\|\nabla^{2} \widetilde{\mathbf{u}}\right\|^{1 / 2}\|\widetilde{\mathbf{u}}\|_{3}^{1 / 2} \leq C\|\widetilde{\mathbf{u}}\|_{3}^{2} .
$$

Using these results in (5.7) we obtain

$$
\|\mathbf{F}\|_{1} \leq C\left(\|\widetilde{\theta}\|_{2}\|\widetilde{\mathbf{B}}\|_{2}+\|\widetilde{\mathbf{u}}\|_{3}^{2}\right)+\|\mathbf{b}\|_{1},
$$


which by (4.20) gives

$$
\|\mathbf{F}\|_{1}^{2} \leq C\left(\|\widetilde{\theta}\|_{2}^{4}+\|\widetilde{\mathbf{B}}\|_{2}^{4}+\|\widetilde{\mathbf{u}}\|_{3}^{4}\right)+\|\mathbf{b}\|_{1}^{2} .
$$

Consequently,

$$
\begin{aligned}
\|\mathbf{F}\|_{L^{2}\left(0, T ; H^{1}\right)}^{2} \leq & C\left(\|\widetilde{\theta}\|_{L^{4}\left(0, T ; H^{2}\right)}^{4}+\|\widetilde{\mathbf{B}}\|_{L^{4}\left(0, T ; H^{2}\right)}^{4}+\|\widetilde{\mathbf{u}}\|_{L^{4}\left(0, T ; H^{3}\right)}^{4}\right)+\|\mathbf{b}\|_{L^{2}\left(0, T ; H^{1}\right)}^{2} \\
\leq & C T\left(\|\widetilde{\theta}\|_{L^{\infty}\left(0, T ; H^{2}\right)}^{4}+\|\widetilde{\mathbf{B}}\|_{L^{\infty}\left(0, T ; H^{2}\right)}^{4}+\|\widetilde{\mathbf{u}}\|_{L^{\infty}\left(0, T ; H^{3}\right)}^{4}\right) \\
& +\|\mathbf{b}\|_{L^{2}\left(0, T ; H^{1}\right)}^{2} \\
\leq & C T\left(K_{1}^{2}+K_{2}^{2}\right)+\|\mathbf{b}\|_{L^{2}\left(0, T ; H^{1}\right)}^{2} .
\end{aligned}
$$

Next, taking the time derivative of $\mathbf{F}$ we obtain

$$
\mathbf{F}_{t}=C_{1}\left(\widetilde{\mathbf{B}}_{t} \nabla \widetilde{\theta}+\widetilde{\mathbf{B}} \nabla \widetilde{\theta}_{t}+\widetilde{\theta}_{t} \operatorname{div} \widetilde{\mathbf{B}}+\widetilde{\theta} \operatorname{div} \widetilde{\mathbf{B}}_{t}\right)-\mathcal{P}\left[\widetilde{\mathbf{u}}_{t} \cdot \nabla\right] \widetilde{\mathbf{u}}-\mathcal{P}[\widetilde{\mathbf{u}} \cdot \nabla] \widetilde{\mathbf{u}}_{t}+\mathbf{b}_{t}
$$

Thus, following the steps leading to (5.8), it follows that

$$
\left\|\mathbf{F}_{t}\right\|_{L^{2}\left(0, T ; H^{-1}\right)}^{2} \leq C T\left[K_{3}^{2}+K_{1}^{2}+K_{2}^{2}+K_{3}^{2}\right]+\left\|\mathbf{b}_{t}\right\|_{L^{2}\left(0, T ; H^{-1}\right)}^{2} .
$$

Returning to (5.4) and using (5.6)-(5.9), we find that

$$
\begin{aligned}
&\|\widehat{\mathbf{u}}\|_{L^{2}\left(0, T ; H^{3}\right) \cap L^{\infty}(0, T ; D(\mathbb{A}))}^{2}+\left\|\widehat{\mathbf{u}}_{t}\right\|_{L^{2}(0, T ; V) \cap L^{\infty}(0, T ; H)}^{2} \\
& \leq C\left[\left\|\mathbb{A} \mathbf{u}_{0}\right\|^{2}+\|\mathbf{b}\|_{L^{2}\left(0, T ; H^{1}\right)}^{2}+\left\|\mathbf{b}_{t}\right\|_{L^{2}\left(0, T ; H^{-1}\right)}^{2}+\left\|\mathbf{B}_{0}\right\|_{2}^{4}+\left\|\theta_{0}\right\|_{2}^{4}\right. \\
&\left.\quad+\left\|\mathbf{u}_{0}\right\|_{2}^{4}+\|\mathbf{b}(\mathbf{0})\|^{2}\right]+C T\left[K_{1}^{2}+K_{2}^{2}+K_{3}^{2}+K_{3}^{2}\right] .
\end{aligned}
$$

Now from the estimate (4.12),

$$
\|\widehat{\theta}\|_{L^{\infty}\left(0, T ; H^{2}\right)}^{2}+\left\|\widehat{\theta}_{t}\right\|_{L^{\infty}\left(0, T ; L^{2}\right) \cap L^{2}\left(0, T ; H^{1}\right)}^{2} \leq C\left[\|f\|_{H^{1}\left(0, T ; L^{2}(\Omega)\right)}^{2}+\left\|\theta_{0}\right\|_{2}^{2}\right],
$$

with

$$
f=-[\widetilde{\mathbf{u}} \cdot \nabla] \widetilde{\theta}+\left[C_{1} \widetilde{\theta} \widetilde{\mathbf{B}}+2 \nu \widetilde{\mathbf{D}}\right]: \widetilde{\mathbf{D}}+r
$$

But

$$
\|f\| \leq C\left(\|\widetilde{\mathbf{u}}\|_{1}\|\widetilde{\theta}\|_{2}+\|\widetilde{\theta}\|_{2}\|\widetilde{\mathbf{B}}\|_{2}\|\widetilde{\mathbf{u}}\|_{3}+\|\widetilde{\mathbf{u}}\|_{2}^{2}\right)+\|r\| .
$$

Combining (5.11), (5.12) and (4.20), one has

$$
\begin{aligned}
& \|\widehat{\theta}\|_{L^{\infty}\left(0, T ; H^{2}\right)}^{2}+\left\|\widehat{\theta}_{t}\right\|_{L^{\infty}\left(0, T ; L^{2}\right) \cap L^{2}\left(0, T ; H^{1}\right)}^{2} \\
& \quad \leq\left(K_{1}^{2}+K_{2}^{2}+K_{1}+K_{2}\right) T+C\left[\|r\|_{H^{1}\left(0, T ; L^{2}(\Omega)\right)}^{2}+\left\|\theta_{0}\right\|_{2}^{2}\right] .
\end{aligned}
$$


Moving to the transport equation, the estimates (4.18) and (4.19) reads

$$
\left\{\begin{array}{l}
\|\widehat{\mathbf{B}}\|_{L^{\infty}\left(0, T ; H^{2}\right)} \leq\left\|\mathbf{B}_{0}\right\|_{2}, \\
\left\|\widehat{\mathbf{B}}_{t}\right\|_{L^{\infty}\left(0, T ; H^{1}\right)} \leq C\left(\|\widetilde{\mathbf{u}}\|_{L^{1}\left(0, T ; H^{3}\right)}+\|\widetilde{\theta}\|_{L^{1}\left(0, T ; H^{2}\right)}\right)\left\|\mathbf{B}_{0}\right\|_{2} \\
\quad+C\|\widetilde{\theta}\|_{L^{1}\left(0, T ; H^{2}\right)}\left[\left\|\mathbf{B}_{0}\right\|_{2}+\left\|\mathbf{B}_{0}\right\|_{2}^{2}\right]^{1 / 2}\left[\left(\left\|\mathbf{B}_{0}\right\|_{2}^{2}+\left\|\mathbf{B}_{0}\right\|_{2}^{4}\right)\left\|\mathbf{B}_{0}\right\|_{2}\right]^{1 / 2} \\
\quad+C\|\widetilde{\theta}\|_{L^{1}\left(0, T ; H^{2}\right)}\left[\left(\left\|\mathbf{B}_{0}\right\|_{2}^{2}+\left\|\mathbf{B}_{0}\right\|_{2}^{4}\right)\left\|\mathbf{B}_{0}\right\|_{2}\right]^{1 / 2}\left[\left(\left\|\mathbf{B}_{0}\right\|_{2}^{3}+\left\|\mathbf{B}_{0}\right\|_{2}^{6}\right)\left\|\mathbf{B}_{0}\right\|_{2}^{2}\right]^{1 / 2},
\end{array}\right.
$$

only if the following condition is fulfilled:

$$
C\left(\|\widetilde{\mathbf{u}}\|_{L^{1}\left(0, T ; H^{3}\right)}+\|\widetilde{\theta}\|_{L^{1}\left(0, T ; H^{2}\right)}+\|\widetilde{\theta}\|_{L^{2}\left(0, T ; H^{2}\right)}^{2}\right) \leq \frac{1}{\left\|\mathbf{B}_{0}\right\|_{2}^{4}} .
$$

But

$$
\begin{aligned}
& C\left(\|\widetilde{\mathbf{u}}\|_{L^{1}\left(0, T ; H^{3}\right)}+\|\widetilde{\theta}\|_{L^{1}\left(0, T ; H^{2}\right)}+\|\widetilde{\theta}\|_{L^{2}\left(0, T ; H^{2}\right)}^{2}\right) \\
& \quad \leq C T\left(\|\widetilde{\mathbf{u}}\|_{L^{\infty}\left(0, T ; H^{3}\right)}+\|\widetilde{\theta}\|_{L^{\infty}\left(0, T ; H^{2}\right)}+\|\widetilde{\theta}\|_{L^{\infty}\left(0, T ; H^{2}\right)}^{2}\right) \\
& \quad \leq C T\left(K_{1}^{1 / 2}+K_{2}^{1 / 2}+K_{2}\right) .
\end{aligned}
$$

So we require that

$$
C T\left(K_{1}^{1 / 2}+K_{2}^{1 / 2}+K_{2}\right) \leq \frac{1}{\left\|\mathbf{B}_{0}\right\|_{2}^{4}} .
$$

One sees that if (5.15) is satisfied, then we automatically obtain $(5.14)_{1,2}$. The upper bound on $(5.14)_{2}$, can be treated as follows:

$$
\begin{aligned}
&\left\|\widehat{\mathbf{B}}_{t}\right\|_{L^{\infty}\left(0, T ; H^{1}\right)} \\
& \leq C T\left(\|\widetilde{\mathbf{u}}\|_{L^{\infty}\left(0, T ; H^{3}\right)}+\|\widetilde{\theta}\|_{L^{\infty}\left(0, T ; H^{2}\right)}\right)\left\|\mathbf{B}_{0}\right\|_{2} \\
&+C T\|\widetilde{\theta}\|_{L^{\infty}\left(0, T ; H^{2}\right)}\left[\left\|\mathbf{B}_{0}\right\|_{2}+\left\|\mathbf{B}_{0}\right\|_{2}^{2}\right]^{1 / 2}\left[\left(\left\|\mathbf{B}_{0}\right\|_{2}^{2}+\left\|\mathbf{B}_{0}\right\|_{2}^{4}\right)\left\|\mathbf{B}_{0}\right\|_{2}\right]^{1 / 2} \\
&+C T\|\widetilde{\theta}\|_{L^{\infty}\left(0, T ; H^{2}\right)}\left[\left(\left\|\mathbf{B}_{0}\right\|_{2}^{2}+\left\|\mathbf{B}_{0}\right\|_{2}^{4}\right)\left\|\mathbf{B}_{0}\right\|_{2}\right]^{1 / 2}\left[\left(\left\|\mathbf{B}_{0}\right\|_{2}^{3}+\left\|\mathbf{B}_{0}\right\|_{2}^{6}\right)\left\|\mathbf{B}_{0}\right\|_{2}^{2}\right]^{1 / 2} \\
& \leq C T\left(K_{1}^{1 / 2}+K_{2}^{1 / 2}\right)\left\|\mathbf{B}_{0}\right\|_{2} \\
&+C T K_{2}^{1 / 2}\left[\left\|\mathbf{B}_{0}\right\|_{2}+\left\|\mathbf{B}_{0}\right\|_{2}^{2}\right]^{1 / 2}\left[\left(\left\|\mathbf{B}_{0}\right\|_{2}^{2}+\left\|\mathbf{B}_{0}\right\|_{2}^{4}\right)\left\|\mathbf{B}_{0}\right\|_{2}\right]^{1 / 2} \\
&+C T K_{2}^{1 / 2}\left[\left(\left\|\mathbf{B}_{0}\right\|_{2}^{2}+\left\|\mathbf{B}_{0}\right\|_{2}^{4}\right)\left\|\mathbf{B}_{0}\right\|_{2}\right]^{1 / 2}\left[\left(\left\|\mathbf{B}_{0}\right\|_{2}^{3}+\left\|\mathbf{B}_{0}\right\|_{2}^{6}\right)\left\|\mathbf{B}_{0}\right\|_{2}^{2}\right]^{1 / 2} .
\end{aligned}
$$

Now, following Refs. 7, 23 and 15, choose $T_{0}$ such that:

(i) $C T_{0}\left(K_{1}^{1 / 2}+K_{2}^{1 / 2}+K_{2}\right) \leq\left\|\mathbf{B}_{0}\right\|_{2}^{-4}$,

(ii) the right-hand side of (5.10) is bounded by $K_{1}$,

(iii) the right-hand side of (5.13) is bounded by $K_{2}$,

(iv) the right-hand side of $(5.14)_{1}$ is bounded by $\max \left(K_{1}, K_{2}\right)$,

(v) the right-hand side of (5.16) is bounded by $K_{3}$. 
So, it is clear that $\mathcal{L}\left(R\left(T_{0}\right)\right) \subset R\left(T_{0}\right)$.

Next, we prove that $\mathcal{L}$ is compact. Indeed, with $T_{0}$ given as above, and based on the estimates (5.10), (5.13), (5.14), and the Aubin-Lions compactness theorem, ${ }^{29} R\left(T_{0}\right)$ is compact in $X\left(T_{0}\right)=\mathcal{C}\left(\left[0, T_{0}\right] ; H^{1}(\Omega)^{d}\right) \times \mathcal{C}\left(\left[0, T_{0}\right] ; H^{1}(\Omega)\right) \times$ $\mathcal{C}\left(\left[0, T_{0}\right] ; H^{1}(\Omega)^{d \times d}\right)$. Hence $\mathcal{L}$ is compact.

Finally, we show that $\mathcal{L}$ is continuous in $X\left(T_{0}\right)$. We know that $R\left(T_{0}\right)$ is compact in $X\left(T_{0}\right)$, thus it will be enough to show that $\mathcal{L}$ is continuous on $Y\left(T_{0}\right)=$ $\mathcal{C}\left(\left[0, T_{0}\right] ; L^{2}(\Omega)^{d}\right) \times \mathcal{C}\left(\left[0, T_{0}\right] ; L^{2}(\Omega)\right) \times \mathcal{C}\left(\left[0, T_{0}\right] ; L^{2}(\Omega)^{d \times d}\right)$.

For that purpose, set

$$
\begin{aligned}
\left(\widehat{\mathbf{u}}_{1}, \widehat{\theta}_{1}, \widehat{\mathbf{B}}_{1}\right) & =\mathcal{L}\left(\widetilde{\mathbf{u}}_{1}, \widetilde{\theta}_{1}, \widetilde{\mathbf{B}}_{1}\right),\left(\widehat{\mathbf{u}}_{2}, \widehat{\theta}_{2}, \widehat{\mathbf{B}}_{2}\right)=\mathcal{L}\left(\widetilde{\mathbf{u}}_{2}, \widetilde{\theta}_{2}, \widetilde{\mathbf{B}}_{2}\right), \\
(\widetilde{\mathbf{u}}, \widetilde{\theta}, \widetilde{\mathbf{B}}) & =\left(\widetilde{\mathbf{u}}_{1}, \widetilde{\theta}_{1}, \widetilde{\mathbf{B}}_{1}\right)-\left(\widetilde{\mathbf{u}}_{2}, \widetilde{\theta}_{2}, \widetilde{\mathbf{B}}_{2}\right),(\widehat{\mathbf{u}}, \widehat{\theta}, \widehat{\mathbf{B}})=\left(\widehat{\mathbf{u}}_{1}, \widehat{\theta}_{1}, \widehat{\mathbf{B}}_{1}\right)-\left(\widehat{\mathbf{u}}_{2}, \widehat{\theta}_{2}, \widehat{\mathbf{B}}_{2}\right),
\end{aligned}
$$

where for $i=1,2,\left(\widetilde{\mathbf{u}}_{i}, \widetilde{\theta}_{i}, \widetilde{\mathbf{B}}_{i}\right)$ is an element of $R\left(T_{0}\right)$.

From (5.1) one obtains

$$
\begin{aligned}
\widehat{\mathbf{u}}_{t}+\widehat{A} \widehat{\mathbf{u}} & =\mathbf{F}\left(\widetilde{\mathbf{u}}_{1}, \widetilde{\theta}_{1}, \widetilde{\mathbf{B}}_{1}\right)-\mathbf{F}\left(\widetilde{\mathbf{u}}_{2}, \widetilde{\theta}_{2}, \widetilde{\mathbf{B}}_{2}\right), \quad \text { a.e. for } t \in(0, T), \\
\widehat{\mathbf{u}}(0) & =0
\end{aligned}
$$

which, by taking the $L^{2}$ inner product of the main equation with $\widehat{\mathbf{u}}$ gives

$$
\begin{aligned}
\frac{1}{2} \frac{d}{d t}\|\widehat{\mathbf{u}}(t)\|^{2}+\|\nabla \widehat{\mathbf{u}}(t)\|^{2}= & C_{1}\left(\operatorname{div}\left(\widetilde{\theta}_{2}(t) \widetilde{\mathbf{B}}(t)+\widetilde{\theta}(t) \widetilde{\mathbf{B}}_{1}(t)\right), \widehat{\mathbf{u}}(t)\right) \\
& -\left([\widetilde{\mathbf{u}}(t) \cdot \nabla] \widetilde{\mathbf{u}}_{1}(t), \widehat{\mathbf{u}}(t)\right)-\left(\left[\widetilde{\mathbf{u}}_{2}(t) \cdot \nabla\right] \widetilde{\mathbf{u}}(t), \widehat{\mathbf{u}}(t)\right) \\
= & -C_{1}\left(\widetilde{\theta}_{2}(t) \widetilde{\mathbf{B}}(t)+\widetilde{\theta}(t) \widetilde{\mathbf{B}}_{1}(t), \nabla \widehat{\mathbf{u}}(t)\right) \\
& -\left([\widetilde{\mathbf{u}}(t) \cdot \nabla] \widetilde{\mathbf{u}}_{1}(t), \widehat{\mathbf{u}}(t)\right)-\left(\left[\widetilde{\mathbf{u}}_{2}(t) \cdot \nabla\right] \widetilde{\mathbf{u}}(t), \widehat{\mathbf{u}}(t)\right) \\
\leq & C\left\|\widetilde{\theta}_{2}(t)\right\|_{2}\|\widetilde{\mathbf{B}}(t)\|\|\nabla \widehat{\mathbf{u}}(t)\|+C\left\|\widetilde{\mathbf{B}}_{1}(t)\right\|_{2}\|\widetilde{\theta}(t)\|\|\nabla \widehat{\mathbf{u}}(t)\| \\
& +\left\|[\widetilde{\mathbf{u}}(t) \cdot \nabla] \widetilde{\mathbf{u}}_{1}(t)\right\|\|\widehat{\mathbf{u}}(t)\|+\left\|\left[\widetilde{\mathbf{u}}_{2}(t) \cdot \nabla\right] \widetilde{\mathbf{u}}(t)\right\|\|\widehat{\mathbf{u}}(t)\| \\
\leq & C\left\|\widetilde{\theta}_{2}(t)\right\|\left\|_{2}\right\| \widetilde{\mathbf{B}}(t)\|\| \nabla \widehat{\mathbf{u}}(t)\|+C\| \widetilde{\mathbf{B}}_{1}(t)\left\|_{2}\right\| \widetilde{\theta}(t)\|\| \nabla \widehat{\mathbf{u}}(t) \| \\
& +C\|\nabla \widetilde{\mathbf{u}}(t)\|\left\|\widetilde{\mathbf{u}}_{1}(t)\right\|\left\|_{2}\right\| \widehat{\mathbf{u}}(t)\|+C\| \widetilde{\mathbf{u}}_{2}(t)\left\|_{2}\right\| \nabla \widetilde{\mathbf{u}}(t)\|\| \widehat{\mathbf{u}}(t) \| .
\end{aligned}
$$

Using the Poincaré's and Young's inequalities, (4.20) leads to

$$
\begin{aligned}
\frac{d}{d t}\|\widehat{\mathbf{u}}(t)\|^{2}+C\|\nabla \widehat{\mathbf{u}}(t)\|^{2} \leq & C\left\|\widetilde{\theta}_{2}(t)\right\|_{2}^{2}\|\widetilde{\mathbf{B}}(t)\|^{2}+C\left\|\widetilde{\mathbf{B}}_{1}(t)\right\|_{2}^{2}\|\widetilde{\theta}(t)\|^{2} \\
& +C\left(\left\|\widetilde{\mathbf{u}}_{1}(t)\right\|_{2}^{2}+\left\|\widetilde{\mathbf{u}}_{2}(t)\right\|_{2}^{2}\right)\|\nabla \widetilde{\mathbf{u}}(t)\|^{2}
\end{aligned}
$$

Therefore

$$
\|\widehat{\mathbf{u}}(t)\| \leq C\|\widetilde{\mathbf{B}}\|_{\mathcal{C}\left(\left[0, T_{0}\right] ; L^{2}\right)}+C\|\widetilde{\theta}\|_{\mathcal{C}\left(\left[0, T_{0}\right] ; L^{2}\right)}+C\|\widetilde{\mathbf{u}}\|_{\mathcal{C}\left(\left[0, T_{0}\right] ; H^{1}\right)} .
$$


Next, from (5.2), one obtains

$$
\begin{aligned}
\widehat{\theta}_{t}-\kappa \Delta \widehat{\theta} & =f\left(\widetilde{\mathbf{u}}_{1}, \widetilde{\theta}_{1}, \widetilde{\mathbf{B}}_{1}\right)-f\left(\widetilde{\mathbf{u}}_{2}, \widetilde{\theta}_{2}, \widetilde{\mathbf{B}}_{2}\right), \quad \text { a.e. in }(0, T), \\
\theta(0) & =0 .
\end{aligned}
$$

Now, proceeding as before, one gets

$$
\begin{aligned}
\frac{1}{2} \frac{d}{d t}\|\widehat{\theta}(t)\|^{2}+\|\nabla \widehat{\theta}(t)\|^{2} \\
\leq C\|\widetilde{\mathbf{u}}(t)\|_{1}\left\|\widetilde{\theta}_{1}(t)\right\|_{2}\|\nabla \widehat{\theta}(t)\|+C\left\|\widetilde{\mathbf{u}}_{2}(t)\right\|_{2}\|\nabla \widetilde{\theta}(t)\|\|\nabla \widehat{\theta}(t)\| \\
\quad+C\|\nabla \widetilde{\theta}(t)\|\|\nabla \widehat{\theta}(t)\|\left\|\widetilde{\mathbf{u}}_{1}(t)\right\|_{3}\left\|\widetilde{\mathbf{B}}_{1}(t)\right\|_{2}+C\|\widetilde{\mathbf{B}}(t)\|_{1}\|\nabla \widehat{\theta}(t)\|\left\|\widetilde{\mathbf{u}}_{1}(t)\right\|_{3}\left\|\widetilde{\theta}_{2}(t)\right\|_{2} \\
\quad+C\|\widetilde{\mathbf{u}}(t)\|_{1}\|\nabla \widehat{\theta}(t)\|\left\|\widetilde{\theta}_{2}(t)\right\|_{2}\left\|\widetilde{\mathbf{B}}_{2}(t)\right\|_{2} \\
\quad+C\left(\left\|\widetilde{\mathbf{u}}_{1}(t)\right\|_{3}+\left\|\widetilde{\mathbf{u}}_{1}(t)\right\|_{3}\right)\|\widetilde{\mathbf{u}}(t)\|_{1}\|\nabla \widehat{\theta}(t)\|,
\end{aligned}
$$

from which one obtains

$$
\begin{aligned}
\frac{d}{d t} \| \widehat{\theta}(t) & \left\|^{2}+C\right\| \nabla \widehat{\theta}(t) \|^{2} \\
\leq & C\|\widetilde{\mathbf{u}}(t)\|_{1}^{2}\left\|\widetilde{\theta}_{1}(t)\right\|_{2}^{2}+C\left\|\widetilde{\mathbf{u}}_{2}(t)\right\|_{2}^{2}\|\nabla \widetilde{\theta}(t)\|^{2} \\
& +C\|\nabla \widetilde{\theta}(t)\|^{2}\left\|\widetilde{\mathbf{u}}_{1}(t)\right\|_{3}^{2}\left\|\widetilde{\mathbf{B}}_{1}(t)\right\|_{2}^{2}+C\|\widetilde{\mathbf{B}}(t)\|_{1}^{2}\left\|\widetilde{\mathbf{u}}_{1}(t)\right\|_{3}^{2}\left\|\widetilde{\theta}_{2}(t)\right\|_{2}^{2} \\
& +C\|\widetilde{\mathbf{u}}(t)\|_{1}^{2}\left\|\widetilde{\theta}_{2}(t)\right\|_{2}^{2}\left\|\widetilde{\mathbf{B}}_{2}(t)\right\|_{2}^{2}+C\left(\left\|\widetilde{\mathbf{u}}_{1}(t)\right\|_{3}^{2}+\left\|\widetilde{\mathbf{u}}_{1}(t)\right\|_{3}^{2}\right)\|\widetilde{\mathbf{u}}(t)\|_{1}^{2} .
\end{aligned}
$$

Thus

$$
\|\widehat{\theta}(t)\| \leq C\|\widetilde{\mathbf{u}}\|_{\mathcal{C}\left(\left[0, T_{0}\right] ; H^{1}\right)}+C\|\widetilde{\theta}\|_{\mathcal{C}\left(\left[0, T_{0}\right] ; H^{1}\right)}+C\|\widetilde{\mathbf{B}}\|_{\mathcal{C}\left(\left[0, T_{0}\right] ; H^{1}\right)} .
$$

Finally, from (4.13), one obtains the equations

$$
\begin{aligned}
\widehat{\mathbf{B}}_{t}+[\widetilde{\mathbf{u}} \cdot \nabla] \widehat{\mathbf{B}}_{2}+\left[\widetilde{\mathbf{u}}_{1} \cdot \nabla\right] \widehat{\mathbf{B}}= & {\left[\nabla \widetilde{\mathbf{u}}_{1}\right] \widehat{\mathbf{B}}+[\nabla \widetilde{\mathbf{u}}] \widehat{\mathbf{B}}_{2}+\widehat{\mathbf{B}}_{1}[\nabla \widetilde{\mathbf{u}}]^{\mathrm{T}}+\widehat{\mathbf{B}}\left[\nabla \widetilde{\mathbf{u}}_{2}\right]^{\mathrm{T}} } \\
& +\alpha_{2} \widetilde{\theta} \frac{d}{\operatorname{tr} \widehat{\mathbf{B}}_{1}^{-1}} \mathbf{I}+d \alpha_{2} \widetilde{\theta}_{2}(t)\left[\frac{\operatorname{tr}\left[\widehat{\mathbf{B}}_{2}^{-1} \widehat{\mathbf{B}} \widehat{\mathbf{B}}_{1}^{-1}\right]}{\operatorname{tr} \widehat{\mathbf{B}}_{1}^{-1} \operatorname{tr} \widehat{\mathbf{B}}_{2}^{-1}}\right] \mathbf{I} \\
& -\alpha_{2} \widetilde{\theta} \widehat{\mathbf{B}}_{1}-\alpha_{2} \widetilde{\theta_{2}} \widehat{\mathbf{B}}, \quad \text { a.a. } t \in(0, T) \\
\widehat{\mathbf{B}}(\mathbf{0})= & 0 .
\end{aligned}
$$

Hence

$$
\begin{aligned}
\frac{1}{2} \frac{d}{d t}\|\widehat{\mathbf{B}}(t)\|^{2} \leq & C\|\widetilde{\mathbf{u}}(t)\|_{1}\left\|\widehat{\mathbf{B}}_{2}(t)\right\|_{2}\|\widehat{\mathbf{B}}(t)\|+C\left(\left\|\widetilde{\mathbf{u}}_{1}(t)\right\|_{3}+\left\|\widetilde{\mathbf{u}}_{2}(t)\right\|_{3}\right)\|\widehat{\mathbf{B}}(t)\|^{2} \\
& +C\|\widetilde{\mathbf{u}}(t)\|_{1}\|\widehat{\mathbf{B}}(t)\|\left(\left\|\widehat{\mathbf{B}}_{2}(t)\right\|_{2}+\left\|\widehat{\mathbf{B}}_{1}(t)\right\|_{2}\right)+C\|\widetilde{\theta}(t)\|\|\widehat{\mathbf{B}}(t)\| \\
& +C\|\widetilde{\theta}(t)\|_{1}\|\widehat{\mathbf{B}}(t)\|\left\|\widehat{\mathbf{B}}_{1}(t)\right\|_{1} \\
& +C\left\|\widetilde{\theta}_{2}(t)\right\|_{2}\|\widehat{\mathbf{B}}(t)\|^{2}+C\left\|\widehat{\mathbf{B}}_{2}^{-1}\right\|_{2}\|\widehat{\mathbf{B}}\|^{2}\left\|\widehat{\mathbf{B}}_{1}^{-1}\right\|\left\|\widetilde{\theta}_{2}\right\|_{2},
\end{aligned}
$$


which gives

$$
\frac{d}{d t}\|\widehat{\mathbf{B}}(t)\|^{2}-C\|\widehat{\mathbf{B}}(t)\|^{2} \leq C\|\widetilde{\mathbf{u}}(t)\|_{1}^{2}+C\|\widetilde{\theta}(t)\|_{1}^{2}
$$

Then

$$
\|\widehat{\mathbf{B}}(t)\| \leq C\|\widetilde{\mathbf{u}}\|_{\mathcal{C}\left(\left[0, T_{0}\right] ; H^{1}\right)}+C\|\widetilde{\theta}\|_{\mathcal{C}\left(\left[0, T_{0}\right], H^{1}\right)} .
$$

From (5.17)-(5.19), one can conclude that $\mathcal{L}$ is continuous on $Y\left(T_{0}\right)=$ $\mathcal{C}\left(\left[0, T_{0}\right] ; L^{2}(\Omega)^{d}\right) \times \mathcal{C}\left(\left[0, T_{0}\right] ; L^{2}(\Omega)\right) \times \mathcal{C}\left(\left[0, T_{0}\right] ; L^{2}(\Omega)^{d \times d}\right)$.

We can then conclude that the mapping $\mathcal{L}$ has a fixed point $(\mathbf{u}, \theta, \mathbf{B})$, say. The pressure is obtained in the standard way (see Ref. 18), and it is unique up to an additive constant.

To obtain the regularity required for the pressure, consider the u-equation of (3.3), that is,

$$
\left\{\begin{array}{l}
-\nu \Delta \mathbf{u}+\operatorname{grad} p=-\left[\mathbf{u}_{t}+[\mathbf{u} \cdot \nabla] \mathbf{u}\right]+\alpha_{1}(\mathbf{B} \nabla \theta+\theta \operatorname{div} \mathbf{B})+\mathbf{b}, \\
\operatorname{div} \mathbf{u}=0 \\
\left.\mathbf{u}\right|_{\partial \Omega}=0
\end{array}\right.
$$

Using the regularity result for the steady Stokes problem (see Lemma 4.1), we have

$$
\begin{aligned}
\|\mathbf{u}\|_{3}+\|\nabla p\|_{1} & \leq C\|\mathbf{b}\|_{1}+C\|\operatorname{div}(\theta \mathbf{B})\|_{1}+C\left\|\mathbf{u}_{t}\right\|_{1}+C\|(\mathbf{u} \cdot \nabla) \mathbf{u}\|_{1} \\
& \leq C\|\mathbf{b}\|_{1}+C\|\theta\|_{2}\|\mathbf{B}\|_{2}+C\left\|\nabla \mathbf{u}_{t}\right\|+C\|(\mathbf{u} \cdot \nabla) \mathbf{u}\|_{1} .
\end{aligned}
$$

But

$$
\|[\mathbf{u} \cdot \nabla] \mathbf{u}\|_{1} \leq C\left(\|\nabla \mathbf{u}\|\left\|\nabla^{2} \mathbf{u}\right\|+\|\nabla \mathbf{u}\|^{1 / 2}\left\|\nabla^{2} \mathbf{u}\right\|^{3 / 2}+\|\nabla \mathbf{u}\|\left\|\nabla^{2} \mathbf{u}\right\|^{1 / 2}\|\mathbf{u}\|_{3}^{1 / 2}\right) .
$$

Thus from (4.4) and (4.20), we obtain

$$
\begin{aligned}
\|\mathbf{u}\|_{3}+\|\nabla p\|_{1} \leq & C\|\mathbf{b}\|_{1}+C\|\theta\|_{2}+C\left\|\nabla \mathbf{u}_{t}\right\| \\
& +C\left(\|\nabla \mathbf{u}\|\|\mathbb{A} \mathbf{u}\|+\|\nabla \mathbf{u}\|^{1 / 2}\|\mathbb{A} \mathbf{u}\|^{3 / 2}+\|\nabla \mathbf{u}\|^{2}\|\mathbb{A} \mathbf{u}\|\right) .
\end{aligned}
$$

On the other hand, $\theta$ satisfies

$$
\left\{\begin{array}{l}
-\kappa \triangle \theta=-\theta_{t}-[\mathbf{u} \cdot \nabla] \theta+\alpha_{1} \theta \mathbf{B}: \mathbf{D}+2 \nu \mathbf{D}: \mathbf{D}+r \text { in } \Omega, \\
\left.\theta\right|_{\partial \Omega}=0 .
\end{array}\right.
$$

Using the regularity of the solution to the Laplace equation (see Ref. 3, p. 181), one has

$$
\begin{aligned}
\|\theta\|_{2} \leq & C\left\|\theta_{t}\right\|+C\|[\mathbf{u} \cdot \nabla] \theta\|+C\|\theta \mathbf{D}\|\|\mathbf{B}\|_{L^{\infty}}+C\|\mathbf{D}\|_{L^{4}}^{2}+C\|r\| \\
\leq & C\left\|\theta_{t}\right\|+C\|\nabla \mathbf{u}\|\|\nabla \theta\|^{1 / 2}\|\theta\|_{2}^{1 / 2}+C\|\nabla \theta\|\|\nabla \mathbf{u}\|^{1 / 2}\|\mathbf{u}\|_{2}^{1 / 2}\|\mathbf{B}\|_{2} \\
& +C\|\nabla \mathbf{u}\|\|\mathbf{u}\|_{2}+C\|r\| .
\end{aligned}
$$


By (4.20) and (4.4), we then find that

$$
\begin{aligned}
\|\theta\|_{2} \leq & C\left\|\theta_{t}\right\|+C\|r\|+C\|\nabla \mathbf{u}\|^{2}\|\nabla \theta\|+C\|\nabla \mathbf{u}\|\|\mathbb{A} \mathbf{u}\| \\
& +C\|\nabla \theta\|\|\nabla \mathbf{u}\|^{1 / 2}\|\mathbf{B}\|_{2}\|\mathbb{A} \mathbf{u}\|^{1 / 2} .
\end{aligned}
$$

Returning to (5.20) with (5.21), we obtain

$$
\begin{aligned}
\|\mathbf{u}\|_{3}+\|\nabla p\|_{1} \leq & C\left(\left\|\theta_{t}\right\|+\|r\|+\|\nabla \mathbf{u}\|^{2}\|\nabla \theta\|+\|\nabla \mathbf{u}\|\|\mathbb{A} \mathbf{u}\|\right. \\
& \left.+\|\nabla \theta\|\|\nabla \mathbf{u}\|^{1 / 2}\|\mathbf{B}\|_{2}\|\mathbb{A} \mathbf{u}\|^{1 / 2}\right)\|\mathbf{B}\|_{2}+C\left\|\nabla \mathbf{u}_{t}\right\| \\
& +C\left(\|\nabla \mathbf{u}\|\|\mathbb{A} \mathbf{u}\|+\|\nabla \mathbf{u}\|^{1 / 2}\|\mathbb{A} \mathbf{u}\|^{3 / 2}+\|\nabla \mathbf{u}\|^{2}\|\mathbb{A} \mathbf{u}\|\right)+C\|\mathbf{b}\|_{1},
\end{aligned}
$$

which shows the desired regularity.

Step 4. Uniqueness of the regular solution. Let $\left(\mathbf{B}^{1}, \mathbf{u}^{1}, \theta^{1}, p^{1}\right)$ and $\left(\mathbf{B}^{2}, \mathbf{u}^{2}, \theta^{2}, p^{2}\right)$ be two solutions to (3.3) that satisfy the regularity properties listed in Theorem 5.1. Set

$$
\left(\mathbf{B}_{12}, \mathbf{u}_{12}, \theta_{12}, p_{12}\right)=\left(\mathbf{B}_{1}-\mathbf{B}_{2}, \mathbf{u}_{1}-\mathbf{u}_{2}, \theta_{1}-\theta_{2}, p_{1}-p_{2}\right) .
$$

Then Eqs. (3.3) yield

$$
\left\{\begin{aligned}
& {\left[\partial_{t} \mathbf{u}_{12}\right.}\left.+\left(\mathbf{u}_{1} \cdot \nabla\right) \mathbf{u}_{12}+\left(\mathbf{u}_{12} \cdot \nabla\right) \mathbf{u}_{2}\right]-\nu \Delta \mathbf{u}_{12}-\alpha_{1} \operatorname{div}\left(\theta_{12} \mathbf{B}_{1}\right) \\
&-\alpha_{1} \operatorname{div}\left(\theta_{2} \mathbf{B}_{12}\right)+\operatorname{grad} p_{12}=0, \\
& \operatorname{div} \mathbf{u}_{12}=0, \quad \mathbf{B}_{12}=\mathbf{B}_{12}^{\mathrm{T}}, \\
& \partial_{t} \mathbf{B}_{12}+\left[\mathbf{u}_{1} \cdot \nabla\right] \mathbf{B}_{12}+\left[\mathbf{u}_{12} \cdot \nabla\right] \mathbf{B}_{2}-\left[\nabla \mathbf{u}_{1}\right] \mathbf{B}_{12} \\
&-\left[\nabla \mathbf{u}_{12}\right] \mathbf{B}_{2}-\mathbf{B}_{12}\left[\nabla \mathbf{u}_{1}\right]-\mathbf{B}_{2}\left[\nabla \mathbf{u}_{12}\right] \\
&= \alpha_{2}\left[\frac{d \mathbf{I}}{\operatorname{tr} \mathbf{B}_{1}^{-1}} \theta_{12}+\frac{d \mathbf{I}}{\operatorname{tr} \mathbf{B}_{1}^{-1}} \theta_{2}-\frac{d \mathbf{I}}{\operatorname{tr} \mathbf{B}_{2}^{-1}} \theta_{2}-\theta_{1} \mathbf{B}_{12}-\theta_{12} \mathbf{B}_{2}\right] \\
& \begin{array}{rl}
\partial_{t} \theta_{12} & \left.+\left(\mathbf{u}_{12} \cdot \nabla\right) \theta_{1}+\left(\mathbf{u}_{2} \cdot \nabla\right) \theta_{12}\right]-\kappa \Delta \theta_{12}
\end{array} \\
&= \alpha_{1} \theta_{12} \mathbf{B}_{1}: \mathbf{D}_{1}+\alpha_{1} \theta_{2} \mathbf{B}_{12}: \mathbf{D}_{2}+\alpha_{1} \theta_{2} \mathbf{B}_{1}: \mathbf{D}_{12} \\
&+2 \nu \mathbf{D}_{1}: \mathbf{D}_{12}+2 \nu \mathbf{D}_{12}: \mathbf{D}_{2}, \\
& \theta_{12} \mid \partial \Omega=0 \quad \text { and }\left.\quad \mathbf{u}_{12}\right|_{\partial \Omega}=0, \\
& \theta_{12}(\mathbf{x}, 0)=0, \quad \mathbf{u}_{12}(\mathbf{x}, 0)=0, \quad \mathbf{B}_{12}(\mathbf{x}, 0)=0 \\
& \operatorname{det} \mathbf{B}_{1}(\mathbf{x}, 0)=1, \quad \operatorname{det} \mathbf{B}_{2}(\mathbf{x}, 0)=1,
\end{aligned}\right.
$$

from which we get, by $L^{2}$-energy estimates,

$$
\begin{aligned}
& \frac{1}{2} \frac{d}{d t}\left\|\mathbf{u}_{12}\right\|^{2}+\nu\left\|\nabla \mathbf{u}_{12}\right\|^{2} \\
& \quad \leq\left\|\mathbf{u}_{12} \cdot \nabla \mathbf{u}_{2}\right\|\left\|\mathbf{u}_{12}\right\|+\alpha_{1}\left\|\theta_{12} \mathbf{B}_{1}\right\|\left\|\mathbf{D}_{12}\right\|+\alpha_{1}\left\|\theta_{2} \mathbf{B}_{12}\right\|\left\|\mathbf{D}_{12}\right\| \\
& \quad \leq C\left\|\nabla \mathbf{u}_{12}\right\|^{2}\left\|\mathbf{u}_{2}\right\|_{2}+C\left\|\theta_{12}\right\|\left\|\mathbf{B}_{1}\right\|_{2}\left\|\nabla \mathbf{u}_{12}+C\right\| \theta_{2}\left\|_{2}\right\| \mathbf{B}_{12}\|\| \nabla \mathbf{u}_{12} \| .
\end{aligned}
$$


Similarly, we obtain

$$
\begin{aligned}
\frac{1}{2} \frac{d}{d t}\left\|\theta_{12}\right\|^{2}+\kappa\left\|\nabla \theta_{12}\right\|^{2} \\
\leq C\left\|\nabla \mathbf{u}_{12}\right\|\left\|\theta_{1}\right\|_{2}\left\|\theta_{12}\right\|+C\left\|\theta_{12}\right\|\left\|\mathbf{B}_{1}\right\|_{2}\left\|\mathbf{u}_{1}\right\|_{2}\left\|\theta_{12}\right\| \\
\quad+C\left\|\theta_{2}\right\|_{2}\left\|\mathbf{B}_{12}\right\|\left\|\mathbf{u}_{2}\right\|_{2}\left\|\theta_{12}\right\|+C\left\|\theta_{2}\right\|_{2}\left\|\nabla \mathbf{u}_{12}\right\|\left\|\mathbf{B}_{1}\right\|_{2}\left\|\theta_{12}\right\| \\
\quad+C\left\|\mathbf{u}_{1}\right\|_{2}\left\|\nabla \mathbf{u}_{12}\right\|\left\|\theta_{12}\right\|+2 C \nu\left\|\nabla \mathbf{u}_{12}\right\|\left\|\mathbf{u}_{2}\right\|_{2}\left\|\theta_{12}\right\| .
\end{aligned}
$$

Finally,

$$
\begin{aligned}
& \frac{1}{2} \frac{d}{d t}\left\|\mathbf{B}_{12}\right\|^{2} \\
&=-\left(\left[\mathbf{u}_{12} \cdot \nabla\right] \mathbf{B}_{2}, \mathbf{B}_{12}\right)+\left(\left[\nabla \mathbf{u}_{1}\right] \mathbf{B}_{12}, \mathbf{B}_{12}\right) \\
&+\left(\left[\nabla \mathbf{u}_{12}\right] \mathbf{B}_{2}, \mathbf{B}_{12}\right)+\left(\mathbf{B}_{12}\left[\nabla \mathbf{u}_{1}\right], \mathbf{B}_{12}\right)+\left(\mathbf{B}_{2}\left[\nabla \mathbf{u}_{12}\right], \mathbf{B}_{12}\right) \\
&+\alpha_{2}\left(\left[\frac{d \mathbf{I}}{\operatorname{tr} \mathbf{B}_{1}^{-1}} \theta_{12}+d \mathbf{I} \frac{\operatorname{tr}\left(\mathbf{B}_{2}^{-1}-\mathbf{B}_{1}^{-1}\right)}{\operatorname{tr} \mathbf{B}_{1}^{-1} \operatorname{tr} \mathbf{B}_{2}^{-1}} \theta_{2}-\theta_{1} \mathbf{B}_{12}-\theta_{12} \mathbf{B}_{2}\right], \mathbf{B}_{12}\right) \\
& \leq C\left\|\nabla \mathbf{u}_{12}\right\|\left\|\mathbf{B}_{2}\right\|_{2}\left\|\mathbf{B}_{12}\right\|+c\left\|\mathbf{u}_{1}\right\|_{2}\left\|\mathbf{B}_{12}\right\|^{2} \\
&+\alpha_{2}\left(\left[\frac{d \mathbf{I}}{\operatorname{tr} \mathbf{B}_{1}^{-1}} \theta_{12}+d I \frac{\operatorname{tr} \mathbf{B}_{1}^{-1}\left(\mathbf{B}_{1}-\mathbf{B}_{2}\right) \mathbf{B}_{2}^{-1}}{\operatorname{tr} \mathbf{B}_{1}^{-1} \operatorname{tr} \mathbf{B}_{2}^{-1}} \theta_{2}-\theta_{1} \mathbf{B}_{12}-\theta_{12} \mathbf{B}_{2}\right], \mathbf{B}_{12}\right) .
\end{aligned}
$$

But from $(4.31)_{1}$, we readily get

$$
\frac{\operatorname{tr}\left[\mathbf{B}_{1}^{-1}\left(\mathbf{B}_{1}-\mathbf{B}_{2}\right) \mathbf{B}_{2}^{-1}\right]}{\operatorname{tr} \mathbf{B}_{1}^{-1} \operatorname{tr} \mathbf{B}_{2}^{-1}} \leq\left|\mathbf{B}_{1}-\mathbf{B}_{2}\right|=\left|\mathbf{B}_{12}\right| .
$$

Thus

$$
\begin{aligned}
\frac{1}{2} \frac{d}{d t}\left\|\mathbf{B}_{12}\right\|^{2} \leq & C\left\|\nabla \mathbf{u}_{12}\right\|\left\|\mathbf{B}_{2}\right\|_{2}\left\|\mathbf{B}_{12}\right\|+C\left\|\mathbf{u}_{1}\right\|_{2}\left\|\mathbf{B}_{12}\right\|^{2} \\
& +C_{2}\left(d I\left|\theta_{12}\right|+d I\left|\mathbf{B}_{12}\left\|\theta_{2}|+| \theta_{1}\right\| \mathbf{B}_{12}\right|+\left|\theta_{12} \| \mathbf{B}_{2}\right|,\left|\mathbf{B}_{12}\right|\right) \\
\leq & C\left\|\nabla \mathbf{u}_{12}\right\|\left\|\mathbf{B}_{2}\right\|_{2}\left\|\mathbf{B}_{12}\right\|+C\left\|\mathbf{u}_{1}\right\|_{2}\left\|\mathbf{B}_{12}\right\|^{2}+C\left\|\theta_{12}\right\|\left\|\mathbf{B}_{12}\right\| \\
& +C\left\|\mathbf{B}_{12}\right\|^{2}\left\|\theta_{2}\right\|_{2}+C\left\|\theta_{1}\right\|_{2}\left\|\mathbf{B}_{12}\right\|^{2}+c\left\|\theta_{12}\right\|\left\|\mathbf{B}_{12}\right\|\left\|\mathbf{B}_{2}\right\| .
\end{aligned}
$$

Now, putting together (5.22)-(5.24), the regularity of $\mathbf{u}_{i}, \theta_{i}, \mathbf{B}_{i}$ derived in Step 3, and using (4.20), we obtain

$$
\begin{aligned}
& \frac{d}{d t}\left[\left\|\mathbf{u}_{12}\right\|^{2}+\left\|\theta_{12}\right\|^{2}+\left\|\mathbf{B}_{12}\right\|^{2}\right]+C\left\|\nabla \theta_{12}\right\|^{2}+C\left\|\nabla \mathbf{u}_{12}\right\|^{2} \\
& \leq C\left[\left\|\mathbf{u}_{12}\right\|^{2}+\left\|\theta_{12}\right\|^{2}+\left\|\mathbf{B}_{12}\right\|^{2}\right] .
\end{aligned}
$$

It follows from Gronwall's lemma that $\left\|\mathbf{u}_{12}\right\|^{2}+\left\|\theta_{12}\right\|^{2}+\left\|\mathbf{B}_{12}\right\|^{2}=0$ a.e. in $\left[0, T_{0}\right]$. The uniqueness of the solutions in the function class described in Theorem 5.1 follows. 


\section{Global Existence of Solutions for Small Data}

In this section we shall prove that the local solution constructed in Theorem 5.1 is global provided that the data are small. Once more, the approach used here follows closely the work of Guillopé and Saut. ${ }^{9}$ The key point in this approach is to derive a new differential inequality (see (6.18)) for the solution constructed in Theorem 5.1 that is valid for all time. The method employed here differs from that used in Ref. 17 because the deformation tensor in the intermediate state $\mathbf{B}$ is not defined via the gradient of a flow mapping.

We claim that

Theorem 6.1. Under the assumptions of Theorem 5.1, assume in addition that

$$
\mathbf{b}_{t} \in L^{2}\left(\mathbb{R}^{+} ; H^{-1}(\Omega)^{d}\right), \quad \mathbf{b} \in L^{2}\left(\mathbb{R}^{+} ; H^{1}(\Omega)^{d}\right), r \in H^{1}\left(\mathbb{R}^{+} ; L^{2}(\Omega)\right),
$$

are small enough in their respective norms.

Then the solution constructed in Theorem 5.1 is global with

(a) $\mathbf{B} \in \mathcal{C}_{b}\left(\mathbb{R}^{+} ; X \cap H^{2}(\Omega)^{d \times d}\right), \mathbf{B}_{t} \in \mathcal{C}_{b}\left(\mathbb{R}^{+} ; H^{1}(\Omega)^{d \times d}\right) \cap L_{\text {loc }}^{2}\left(\mathbb{R}^{+} ; H^{1}(\Omega)^{d \times d}\right)$,

(b) $\mathbf{u} \in \mathcal{C}_{b}\left(\mathbb{R}^{+} ; D(\mathbb{A})\right) \cap L_{\text {loc }}^{2}\left(\mathbb{R}^{+} ; H^{3}(\Omega)^{d}\right)$ with $\mathbf{u}_{t} \in \mathcal{C}_{b}\left(\mathbb{R}^{+} ; H\right) \cap L_{\text {loc }}^{2}\left(\mathbb{R}^{+} ; V\right)$,

(c) $p \in L_{\text {loc }}^{2}\left(\mathbb{R}^{+} ; H^{2}(\Omega)\right)$, and

(d) $\theta \in \mathcal{C}_{b}\left(\mathbb{R}^{+} ; H^{2}(\Omega) \cap H_{0}^{1}(\Omega)\right)$, with $\theta_{t} \in \mathcal{C}_{b}\left(\mathbb{R}^{+} ; L^{2}(\Omega)\right) \cap L_{\text {loc }}^{2}\left(\mathbb{R}^{+} ; H_{0}^{1}(\Omega)\right)$.

Proof. The proof proceeds in two steps.

\section{Step 1. New differential inequality}

Let $(\mathbf{u}, \theta, \mathbf{B}, p)$ be the solution of (3.3) given in Theorem 5.1.

We start by deriving differential inequalities for $\mathbf{u}, \theta, \mathbf{B}$, and $\mathbf{u}_{t}, \theta_{t}, \mathbf{B}_{t}$.

First, replacing $(\widetilde{\mathbf{u}}, \widetilde{\theta}, \widetilde{\mathbf{B}})$ by $(\mathbf{u}, \theta, \mathbf{B})$ in $(4.39)$, one obtains

$$
\frac{d}{d t}\|\mathbf{B}(t)\|_{2}^{2} \leq C\left(\|\mathbf{u}(t)\|_{3}+\|\theta(t)\|_{2}+\|\theta(t)\|_{2}^{2}\right)\left(1+\|\mathbf{B}(t)\|_{2}^{2}\right)^{5} .
$$

By applying $\partial_{t}$ to the $\mathbf{B}$-equation of (3.3), and taking the $L^{2}$ inner product of the resulting equation with $\mathbf{B}_{t}$, one has

$$
\begin{aligned}
\frac{1}{2} \frac{d}{d t}\left\|\mathbf{B}_{t}(t)\right\|^{2}= & -\left(\left(\mathbf{u}_{t} \cdot \nabla\right) \mathbf{B}, \mathbf{B}_{t}\right)+\left(\left([\nabla \mathbf{u}] \mathbf{B}+\mathbf{B}[\nabla \mathbf{u}]^{\mathrm{T}}\right)_{t}, \mathbf{B}_{t}\right)-\alpha_{2}\left(\theta_{t} \mathbf{B}, \mathbf{B}_{t}\right) \\
& +\alpha_{2} d\left(\frac{\theta_{t}}{\operatorname{tr} \mathbf{B}^{-1}}, \operatorname{tr} \mathbf{B}_{t}\right)+\alpha_{2} d\left(\frac{\operatorname{tr}\left[\mathbf{B}^{-1} \mathbf{B}_{t} \mathbf{B}^{-1}\right]}{\left[\operatorname{tr} \mathbf{B}^{-1}\right]^{2}}, \theta \mathbf{B}_{t}\right)-\alpha_{2}\left(\theta,\left|\mathbf{B}_{t}\right|^{2}\right) .
\end{aligned}
$$

But,

$$
\begin{aligned}
\left(\left(\mathbf{u}_{t} \cdot \nabla\right) \mathbf{B}, \mathbf{B}_{t}\right) & \leq\left\|\left(\mathbf{u}_{t} \cdot \nabla\right) \mathbf{B}\right\|\left\|\mathbf{B}_{t}\right\| \leq C\left\|\nabla \mathbf{u}_{t}\right\|\|\mathbf{B}\|_{2}\left\|\mathbf{B}_{t}\right\|, \\
\left(\left(\nabla \mathbf{u B}+\mathbf{B}[\nabla \mathbf{u}]^{\mathrm{T}}\right)_{t}, \mathbf{B}_{t}\right) & \leq\left\|\left(\nabla \mathbf{u B}+\mathbf{B}[\nabla \mathbf{u}]^{\mathrm{T}}\right)_{t}\right\|\left\|\mathbf{B}_{t}\right\| \\
& \leq 2\left\{\left\|\nabla \mathbf{u}_{t}\right\|\|\mathbf{B}\|_{L^{\infty}}+\left\|\mathbf{B}_{t}\right\|\|\nabla \mathbf{u}\|_{L^{\infty}}\right\}\left\|\mathbf{B}_{t}\right\| \\
& \leq C\left\{\left\|\nabla \mathbf{u}_{t}\right\|\left\|\mathbf{B}_{t}\right\|\|\mathbf{B}\|_{2}+\left\|\mathbf{B}_{t}\right\|^{2}\|\nabla \mathbf{u}\|_{1}^{1 / 2}\|\nabla \mathbf{u}\|_{2}^{1 / 2}\right\},
\end{aligned}
$$




$$
\begin{gathered}
\left(\theta_{t} \mathbf{B}, \mathbf{B}_{t}\right) \leq\|\mathbf{B}\|_{L^{\infty}}\left\|\theta_{t}\right\|\left\|\mathbf{B}_{t}\right\| \leq C\|\mathbf{B}\|_{2}\left\|\theta_{t}\right\|\left\|\mathbf{B}_{t}\right\|, \\
\left(\frac{\theta_{t}}{\operatorname{tr} \mathbf{B}^{-1}}, \operatorname{tr} \mathbf{B}_{t}\right) \leq\left(\frac{1}{\operatorname{tr} \mathbf{B}^{-1}}\left|\theta_{t}\right|,\left|\operatorname{tr} \mathbf{B}_{t}\right|\right) \leq \frac{1}{d}\left(\left|\theta_{t}\right|,\left|\operatorname{tr} \mathbf{B}_{t}\right|\right) \leq C\left\|\theta_{t}\right\|\left\|\mathbf{B}_{t}\right\|, \\
\left(\frac{\operatorname{tr}\left[\mathbf{B}^{-1} \mathbf{B}_{t} \mathbf{B}^{-1}\right]}{\left[\operatorname{tr} \mathbf{B}^{-1}\right]^{2}}, \theta \mathbf{B}_{t}\right) \leq C\left(\left|\mathbf{B}_{t}\right|^{2}, \theta\right) \leq C\|\theta\|_{L^{\infty}}\left\|\mathbf{B}_{t}\right\|^{2} \leq C\|\nabla \theta\|^{1 / 2}\|\theta\|_{2}^{1 / 2}\left\|\mathbf{B}_{t}\right\|^{2}, \\
\left(\theta,\left|\mathbf{B}_{t}\right|^{2}\right) \leq\|\theta\|_{L^{\infty}}\left\|\mathbf{B}_{t}\right\|^{2} \leq C\|\nabla \theta\|^{1 / 2}\|\theta\|_{2}^{1 / 2}\left\|\mathbf{B}_{t}\right\|^{2} .
\end{gathered}
$$

We thus conclude that (after using Lemma 4.1)

$$
\begin{aligned}
\frac{d}{d t}\left\|\mathbf{B}_{t}(t)\right\|^{2} \leq & C\left(\left\|\nabla \mathbf{u}_{t}\right\|+\left\|\theta_{t}\right\|\right)\left\|\mathbf{B}_{t}\right\|\|\mathbf{B}\|_{2} \\
& +C\left\|\theta_{t}\right\|\left\|\mathbf{B}_{t}\right\|+C\left(\|\nabla \theta\|^{1 / 2}\|\theta\|_{2}^{1 / 2}+\|\mathbb{A} \mathbf{u}\|^{1 / 2}\|\mathbf{u}\|_{3}^{1 / 2}\right)\left\|\mathbf{B}_{t}\right\|^{2},
\end{aligned}
$$

which, with (6.1) and (4.20) yields

$$
\begin{aligned}
\frac{d}{d t}\left[\|\mathbf{B}(t)\|_{2}^{2}+\left\|\mathbf{B}_{t}(t)\right\|^{2}\right] \leq & C\left(\|\mathbf{u}(t)\|_{3}+\left\|\nabla \mathbf{u}_{t}\right\|+\left\|\theta_{t}\right\|+\|\theta(t)\|_{2}\right. \\
& \left.+\|\theta(t)\|_{2}^{2}\right)\left(1+\|\mathbf{B}(t)\|_{2}^{2}\right)^{5}+C\left(\left\|\nabla \mathbf{u}_{t}\right\|+\left\|\theta_{t}\right\|\right. \\
& \left.+\|\nabla \theta\|^{1 / 2}\|\theta\|_{2}^{1 / 2}+\|\mathbb{A} \mathbf{u}\|^{1 / 2}\|\mathbf{u}\|_{3}^{1 / 2}\right)\left(1+\left\|\mathbf{B}_{t}\right\|\right)^{2} .
\end{aligned}
$$

We now turn to the estimates for $\mathbf{u}$. We first take the $L^{2}$-inner product of the $\mathbf{u}$-equation of (3.3) with $\mathbb{A} \mathbf{u}$ to obtain

$$
\begin{aligned}
\frac{1}{2} \frac{d}{d t}\|\nabla \mathbf{u}(t)\|^{2}+\|\mathbb{A} \mathbf{u}\|^{2}= & -((\mathbf{u} \cdot \nabla) \mathbf{u}, \mathbb{A} \mathbf{u})-\alpha_{1}(\operatorname{div}(\theta \mathbf{B}), \mathbb{A} \mathbf{u})-(\mathbf{b}, \mathbb{A} \mathbf{u}) \\
\leq & \|(\mathbf{u} \cdot \nabla) \mathbf{u}\|\|\mathbb{A} \mathbf{u}\|+\alpha_{1}\|\operatorname{div}(\theta \mathbf{B})\|\|\mathbb{A} \mathbf{u}\|+\|\mathbf{b}\|\|\mathbb{A} \mathbf{u}\| \\
\leq & C\|\nabla \mathbf{u}\|^{3 / 2}\left\|\partial^{2} \mathbf{u}\right\|^{1 / 2}\|\mathbb{A} \mathbf{u}\|+\|\mathbf{b}\|\|\mathbb{A} \mathbf{u}\| \\
& +C\left\{\|\mathbf{B}\|_{L^{\infty}}\|\nabla \theta\|+\|\theta\|_{L^{3}}\|\operatorname{div} \mathbf{B}\|_{L^{6}}\right\}\|\mathbb{A} \mathbf{u}\| \\
\leq & C\|\nabla \mathbf{u}\|^{3 / 2}\left\|\partial^{2} \mathbf{u}\right\|^{1 / 2}\|\mathbb{A} \mathbf{u}\|+\|\mathbf{b}\|\|\mathbb{A} \mathbf{u}\| \\
& +C\left\{\|\mathbf{B}\|_{2}\|\nabla \theta\|+\|\theta\|^{1 / 2}\|\nabla \theta\|^{1 / 2}\|\mathbf{B}\|_{2}\right\}\|\mathbb{A} \mathbf{u}\|
\end{aligned}
$$

which, together with Lemma 4.1 and (4.20) gives

$$
\begin{aligned}
& \frac{d}{d t}\|\nabla \mathbf{u}(t)\|^{2}+C\|\mathbb{A} \mathbf{u}\|^{2} \\
& \quad \leq C\|\nabla \mathbf{u}\|^{6}+C\left\{\|\mathbf{B}\|_{2}^{2}\|\nabla \theta\|^{2}+\|\theta\|\|\nabla \theta\|\|\mathbf{B}\|_{2}^{2}\right\}+C\|\mathbf{b}\|^{2} .
\end{aligned}
$$

To derive the estimates concerning the time derivative of the velocity, we first note that $\left.\mathbf{u}_{t}\right|_{\partial \Omega}=0$. Then using Poincaré's inequality, and proceeding as before, we obtain

$$
\begin{aligned}
\frac{1}{2} \frac{d}{d t}\left\|\mathbf{u}_{t}(t)\right\|^{2}+\nu\left\|\nabla \mathbf{u}_{t}\right\|^{2} & =-\left(\left(\mathbf{u}_{t} \cdot \nabla\right) \mathbf{u}, \mathbf{u}_{t}\right)-\alpha_{1}\left((\theta \mathbf{B})_{t}, \nabla \mathbf{u}_{t}\right)+\left(\mathbf{b}_{t}, \mathbf{u}_{t}\right) \\
& \leq\left\|\left(\mathbf{u}_{t} \cdot \nabla\right) \mathbf{u}\right\|\left\|\mathbf{u}_{t}\right\|+\alpha_{1}\left\|(\theta \mathbf{B})_{t}\right\|\left\|\nabla \mathbf{u}_{t}\right\|+\left\|\mathbf{b}_{t}\right\|\left\|\mathbf{u}_{t}\right\|
\end{aligned}
$$




$$
\begin{aligned}
\leq & C\left\|\nabla \mathbf{u}_{t}\right\|\|\nabla \mathbf{u}\|^{1 / 2}\left\|\partial^{2} \mathbf{u}\right\|^{1 / 2}\left\|\mathbf{u}_{t}\right\|+C\left\|\mathbf{b}_{t}\right\|\left\|\nabla \mathbf{u}_{t}\right\| \\
& +C\left\{\left\|\theta_{t}\right\|_{L^{3}}\|\mathbf{B}\|_{L^{6}}+\left\|\mathbf{B}_{t}\right\|\|\theta\|_{L^{\infty}}\right\}\left\|\nabla \mathbf{u}_{t}\right\| \\
\leq & C\left\|\nabla \mathbf{u}_{t}\right\|\|\nabla \mathbf{u}\|^{1 / 2}\left\|\partial^{2} \mathbf{u}\right\|^{1 / 2}\left\|\mathbf{u}_{t}\right\|+C\left\|\mathbf{b}_{t}\right\|\left\|\nabla \mathbf{u}_{t}\right\| \\
& +C\left\{\left\|\theta_{t}\right\|^{1 / 2}\left\|\nabla \theta_{t}\right\|^{1 / 2}\|\mathbf{B}\|_{2}+\left\|\mathbf{B}_{t}\right\|\|\nabla \theta\|^{1 / 2}\|\theta\|_{2}^{1 / 2}\right\}\left\|\nabla \mathbf{u}_{t}\right\|,
\end{aligned}
$$

which with (4.20) gives

$$
\begin{aligned}
\frac{d}{d t}\left\|\mathbf{u}_{t}(t)\right\|^{2}+C\left\|\nabla \mathbf{u}_{t}\right\|^{2} \leq & C\|\nabla \mathbf{u}\|\|\mathbb{A} \mathbf{u}\|\left\|\mathbf{u}_{t}\right\|^{2}+C\left\|\theta_{t}\right\|\left\|\nabla \theta_{t}\right\|\|\mathbf{B}\|_{2}^{2} \\
& +C\left\|\mathbf{B}_{t}\right\|^{2}\|\nabla \theta\|\|\theta\|_{2}+C\left\|\mathbf{b}_{t}\right\|^{2} .
\end{aligned}
$$

Putting together (6.3), (6.4), and using (4.20), one gets

$$
\begin{aligned}
& \frac{d}{d t}\left[\left\|\mathbf{u}_{t}(t)\right\|^{2}+\|\nabla \mathbf{u}(t)\|^{2}\right]+C\left\|\nabla \mathbf{u}_{t}\right\|^{2}+C\|\mathbb{A} \mathbf{u}\|^{2} \\
& \leq C\|\nabla \mathbf{u}\|^{2}\left\|\mathbf{u}_{t}\right\|^{4}+C\left\|\theta_{t}\right\|\left\|\nabla \theta_{t}\right\|\|\mathbf{B}\|_{2}^{2}+C\left\|\mathbf{B}_{t}\right\|^{2}\|\nabla \theta\|\|\theta\|_{2} \\
&+C\|\mathbf{B}\|_{2}^{2}\|\nabla \theta\|^{2}+C\|\theta\|\|\nabla \theta\|\|\mathbf{B}\|_{2}^{2}+C\|\nabla \mathbf{u}\|^{6}+C\|\mathbf{b}\|^{2}+C\left\|\mathbf{b}_{t}\right\|^{2} .
\end{aligned}
$$

Next, we turn our attention to the estimate of $\theta$. We then take the $L^{2}$ inner product of the $\theta$-equation of (3.3), with $-\triangle \theta$, which yields

$$
\begin{aligned}
\frac{1}{2} \frac{d}{d t} \| & \nabla \theta(t)\left\|^{2}+\kappa\right\| \triangle \theta \|^{2} \\
= & -((\mathbf{u} \cdot \nabla) \theta, \triangle \theta)+\alpha_{1}(\theta \mathbf{B}: \mathbf{D}, \triangle \theta)+2 \nu(\mathbf{D}: \mathbf{D}, \triangle \theta)-(r, \triangle \theta) \\
\leq & \|(\mathbf{u} \cdot \nabla) \theta\|\|\triangle \theta\|+C_{1}\|\theta \mathbf{B}: \mathbf{D}\|\|\triangle \theta\|+2 \nu\|\nabla \mathbf{u}: \nabla \mathbf{u}\|\|\triangle \theta\|+\|r\|\|\triangle \theta\| \\
\leq & C\|\nabla \mathbf{u}\|\|\nabla \theta\|^{1 / 2}\|\theta\|_{2}^{1 / 2}\|\triangle \theta\|+C\|\theta\|\|\mathbf{B}\|_{2}\|\mathbf{u}\|_{3}\|\triangle \theta\| \\
& +C\|\nabla \mathbf{u}\|^{1 / 2}\|\nabla \mathbf{u}\|_{1}^{3 / 2}\|\triangle \theta\|+\|r\|\|\triangle \theta\| .
\end{aligned}
$$

With (4.20) and (4.4), this gives

$$
\begin{aligned}
\frac{d}{d t}\|\nabla \theta(t)\|^{2}+C\|\triangle \theta\|^{2} \leq & C\left\{\|\nabla \mathbf{u}\|^{1 / 2}\|\mathbb{A} \mathbf{u}\|^{3 / 2}+\|\nabla \mathbf{u}\|^{2}\|\nabla \theta\|\right\}\|\theta\|_{2} \\
& +C\|\nabla \theta\|^{2}\|\mathbf{B}\|_{2}^{2}\|\mathbf{u}\|_{3}^{2}+C\|r\|^{2} .
\end{aligned}
$$

Finally, we notice that $\left.\theta_{t}\right|_{\partial \Omega}=0$. Applying the time derivative to the $\theta$-equation of (3.3) and taking the $L^{2}$ inner product of the resulting equation with $\theta_{t}$, we obtain

$$
\begin{aligned}
\frac{1}{2} \frac{d}{d t}\left\|\theta_{t}(t)\right\|^{2}+\kappa\left\|\nabla \theta_{t}\right\|^{2} & \\
= & -\left(\left(\mathbf{u}_{t} \cdot \nabla\right) \theta, \theta_{t}\right)+\left(\left(\alpha_{1} \theta \mathbf{B}+2 \nu \mathbf{D}\right)_{t}: \mathbf{D}, \theta_{t}\right) \\
& +\left(\left(\alpha_{1} \theta \mathbf{B}+2 \nu \mathbf{D}\right): \mathbf{D}_{t}, \theta_{t}\right)+\left(r_{t}, \theta_{t}\right)
\end{aligned}
$$




$$
\begin{aligned}
\leq & C\left\|\mathbf{u}_{t}\right\|^{1 / 2}\left\|\nabla \mathbf{u}_{t}\right\|^{1 / 2}\|\nabla \theta\|^{1 / 2}\|\theta\|_{2}^{1 / 2}\left\|\theta_{t}\right\| \\
& +C\left\|\nabla \theta_{t}\right\|^{1 / 2}\|\mathbf{B}\|_{2}\|\mathbf{u}\|_{2}^{1 / 2}\|\mathbf{u}\|_{3}^{1 / 2}\left\|\theta_{t}\right\|^{5 / 4}\left\|\nabla \theta_{t}\right\|^{1 / 4} \\
& +C\|\nabla \theta\|^{1 / 2}\|\theta\|_{2}^{1 / 2}\|\mathbf{u}\|_{2}^{1 / 2}\|\mathbf{u}\|_{3}^{1 / 2}\left\|\mathbf{B}_{t}\right\|\left\|\theta_{t}\right\|+C\left\|\nabla \mathbf{u}_{t}\right\|\|\mathbf{u}\|_{2}^{1 / 2}\|\mathbf{u}\|_{3}^{1 / 2}\left\|\theta_{t}\right\| \\
& +C\|\mathbf{u}\|_{3}\left\|\nabla \mathbf{u}_{t}\right\|\left\|\theta_{t}\right\|+C\|\nabla \theta\|^{1 / 2}\|\theta\|_{2}^{1 / 2}\|\mathbf{B}\|_{2}\left\|\nabla \mathbf{u}_{t}\right\|\left\|\theta_{t}\right\|+\left\|r_{t}\right\|\left\|\theta_{t}\right\|,
\end{aligned}
$$

which by Poincaré's inequality and (4.20) yield;

$$
\begin{aligned}
\frac{d}{d t} \| \theta_{t}(t) & \left\|^{2}+C\right\| \nabla \theta_{t} \|^{2} \\
\leq & C\left\|\mathbf{u}_{t}\right\|\left\|\nabla \mathbf{u}_{t}\right\|\|\nabla \theta\|\|\theta\|_{2}+C\|\mathbf{B}\|_{2}^{4 / 3}\|\mathbf{u}\|_{2}^{2 / 3}\|\mathbf{u}\|_{3}^{2 / 3}\left\|\theta_{t}\right\|^{5 / 3}\left\|\nabla \theta_{t}\right\|^{1 / 3} \\
& +C\|\nabla \theta\|\|\theta\|_{2}\|\mathbf{u}\|_{2}\|\mathbf{u}\|_{3}\left\|\mathbf{B}_{t}\right\|^{2}+C\left\|\nabla \mathbf{u}_{t}\right\|\|\mathbf{u}\|_{2}^{1 / 2}\|\mathbf{u}\|_{3}^{1 / 2}\left\|\theta_{t}\right\| \\
& +C\|\mathbf{u}\|_{3}\left\|\nabla \mathbf{u}_{t}\right\|\left\|\theta_{t}\right\|+C\|\nabla \theta\|^{1 / 2}\|\theta\|_{2}^{1 / 2}\|\mathbf{B}\|_{2}\left\|\nabla \mathbf{u}_{t}\right\|\left\|\theta_{t}\right\|+C\left\|r_{t}\right\|^{2} .
\end{aligned}
$$

Putting together (6.6), (6.7), and (4.4), one obtains

$$
\begin{aligned}
\frac{d}{d t}[\| \nabla & \left.\theta(t)\left\|^{2}+\right\| \theta_{t}(t) \|^{2}\right]+C\left\|\nabla \theta_{t}\right\|^{2}+C\|\triangle \theta\|^{2} \\
\leq & C\left\|\mathbf{u}_{t}\right\|\left\|\nabla \mathbf{u}_{t}\right\|\|\nabla \theta\|\|\theta\|_{2}+C\|\mathbf{B}\|_{2}^{4 / 3}\|\mathbb{A} \mathbf{u}\|^{2 / 3}\|\mathbf{u}\|_{3}^{2 / 3}\left\|\theta_{t}\right\|^{5 / 3}\left\|\nabla \theta_{t}\right\|^{1 / 3} \\
& +C\|\nabla \theta\|\|\theta\|_{2}\|\mathbb{A} \mathbf{u}\|\|\mathbf{u}\|_{3}\left\|\mathbf{B}_{t}\right\|^{2}+C\left\|\nabla \mathbf{u}_{t}\right\|\|\mathbb{A} \mathbf{u}\|^{1 / 2}\|\mathbf{u}\|_{3}^{1 / 2}\left\|\theta_{t}\right\| \\
& +C\|\mathbf{u}\|_{3}\left\|\nabla \mathbf{u}_{t}\right\|\left\|\theta_{t}\right\|+C\|\nabla \theta\|^{1 / 2}\|\theta\|_{2}^{1 / 2}\|\mathbf{B}\|_{2}\left\|\nabla \mathbf{u}_{t}\right\|\left\|\theta_{t}\right\| \\
& +C\left\{\|\nabla \mathbf{u}\|^{1 / 2}\|\mathbb{A} \mathbf{u}\|^{3 / 2}+\|\nabla \mathbf{u}\|^{2}\|\nabla \theta\|\right\}\|\theta\|_{2} \\
& +C\|\nabla \theta\|^{2}\|\mathbf{B}\|_{2}^{2}\|\mathbf{u}\|_{3}^{2}+C\|r\|^{2}+C\left\|r_{t}\right\|^{2} .
\end{aligned}
$$

Performing (6.2), (6.5), and (6.8), one obtains

$$
\begin{aligned}
& \frac{d}{d t}\left[\|\mathbf{B}(t)\|_{2}^{2}+\left\|\mathbf{B}_{t}(t)\right\|^{2}+\left\|\mathbf{u}_{t}(t)\right\|^{2}+\|\nabla \mathbf{u}(t)\|^{2}+\|\nabla \theta(t)\|^{2}+\left\|\theta_{t}(t)\right\|^{2}\right] \\
&+C\left\|\nabla \mathbf{u}_{t}\right\|^{2}+C\|\mathbb{A} \mathbf{u}\|^{2}+C\left\|\nabla \theta_{t}\right\|^{2}+C\|\triangle \theta\|^{2} \\
& \leq C\left(\|\mathbf{u}(t)\|_{3}+\left\|\nabla \mathbf{u}_{t}\right\|+\left\|\theta_{t}\right\|+\left\|\theta_{t}\right\|\left\|\nabla \theta_{t}\right\|+\|\theta(t)\|_{2}+\|\theta(t)\|_{2}^{2}\right. \\
&\left.+\|\nabla \theta\|^{2}\|\mathbf{u}\|_{3}^{2}\right)\left(1+\|\mathbf{B}(t)\|_{2}^{2}\right)^{5}+C\left(\left\|\nabla \mathbf{u}_{t}\right\|+\left\|\theta_{t}\right\|+\|\nabla \theta\|^{1 / 2}\|\theta\|_{2}^{1 / 2}\right. \\
&\left.+\|\mathbb{A} \mathbf{u}\|^{1 / 2}\|\mathbf{u}\|_{3}^{1 / 2}+\|\nabla \theta\|\|\theta\|_{2}\|\mathbb{A} \mathbf{u}\|\|\mathbf{u}\|_{3}+\|\nabla \theta\|\|\theta\|_{2}\right)\left(1+\left\|\mathbf{B}_{t}\right\|\right)^{2} \\
&+C\|\mathbf{u}\|_{3}\left\|\nabla \mathbf{u}_{t}\right\|\left\|\theta_{t}\right\|+C\|\nabla \mathbf{u}\|^{2}\left\|\mathbf{u}_{t}\right\|^{4}+C\left\|\nabla \mathbf{u}_{t}\right\|\|\mathbb{A} \mathbf{u}\|^{1 / 2}\|\mathbf{u}\|_{3}^{1 / 2}\left\|\theta_{t}\right\| \\
&+C\|\nabla \mathbf{u}\|^{6}+C\|\mathbf{B}\|_{2}^{4 / 3}\|\mathbb{A} \mathbf{u}\|^{2 / 3}\|\mathbf{u}\|_{3}^{2 / 3}\left\|\theta_{t}\right\|^{5 / 3}\left\|\nabla \theta_{t}\right\|^{1 / 3}
\end{aligned}
$$




$$
\begin{aligned}
& +C\|\nabla \theta\|^{1 / 2}\|\theta\|_{2}^{1 / 2}\|\mathbf{B}\|_{2}\left\|\nabla \mathbf{u}_{t}\right\|\left\|\theta_{t}\right\|+C\left(\|\nabla \mathbf{u}\|^{1 / 2}\|\mathbb{A} \mathbf{u}\|^{3 / 2}+\|\nabla \mathbf{u}\|^{2}\|\nabla \theta\|\right. \\
& \left.+\left\|\mathbf{u}_{t}\right\|\left\|\nabla \mathbf{u}_{t}\right\|\|\nabla \theta\|\right)\|\theta\|_{2}+C\|\mathbf{b}\|^{2}+C\left\|\mathbf{b}_{t}\right\|^{2}+C\|r\|^{2}+C\left\|r_{t}\right\|^{2}
\end{aligned}
$$

In order to close the estimates in (6.9), it is manifested that one has to estimate $\|\mathbf{u}\|_{3}$ and $\|\theta\|_{2}$. First, from the $\mathbf{u}$-equation of (3.3),

$$
\left\{\begin{array}{l}
-\nu \Delta \mathbf{u}+\operatorname{grad} p=-\left[\mathbf{u}_{t}+[\mathbf{u} \cdot \nabla] \mathbf{u}\right]+\alpha_{1} \operatorname{div}(\theta \mathbf{B})+\mathbf{b}, \\
\operatorname{div} \mathbf{u}=0 \\
\left.\mathbf{u}\right|_{\partial \Omega}=0
\end{array}\right.
$$

Using the regularity result for the steady Stokes problem (see Lemma 4.1), we have

$$
\begin{aligned}
\|\mathbf{u}\|_{3} & \leq C\|\mathbf{b}\|_{1}+c\|\operatorname{div}(\theta \mathbf{B})\|_{1}+C\left\|\mathbf{u}_{t}\right\|_{1}+C\|(\mathbf{u} \cdot \nabla) \mathbf{u}\|_{1} \\
& \leq C\|\mathbf{b}\|_{1}+C\|\theta\|_{2}\|\mathbf{B}\|_{2}+C\left\|\nabla \mathbf{u}_{t}\right\|+C\|(\mathbf{u} \cdot \nabla) \mathbf{u}\|_{1} .
\end{aligned}
$$

But from Step 3 in Sec. 5 one has

$$
\|[\mathbf{u} \cdot \nabla] \mathbf{u}\|_{1} \leq C\left(\|\nabla \mathbf{u}\|\left\|\partial^{2} \mathbf{u}\right\|+\|\nabla \mathbf{u}\|^{1 / 2}\left\|\partial^{2} \mathbf{u}\right\|^{3 / 2}+\|\nabla \mathbf{u}\|\left\|\partial^{2} \mathbf{u}\right\|^{1 / 2}\|\mathbf{u}\|_{3}^{1 / 2}\right),
$$

which together with (4.4) and (4.20) yields

$$
\begin{aligned}
\|\mathbf{u}\|_{3} \leq & C\|\mathbf{b}\|_{1}+C\left\|\nabla \mathbf{u}_{t}\right\|+C\|\nabla \mathbf{u}\|^{2}+C\|\nabla \mathbf{u}\|^{4} \\
& +C\|\theta\|_{2}\|\mathbf{B}\|_{2}+C\|\mathbb{A} \mathbf{u}\|^{2} .
\end{aligned}
$$

Now, projecting the u-equation of (3.3) on $H$, this becomes

$$
\left\{\begin{array}{l}
\mathbb{A} \mathbf{u}=-\mathcal{P}\left[\mathbf{u}_{t}+[\mathbf{u} \cdot \nabla] \mathbf{u}\right]+\alpha_{1} \mathcal{P} \operatorname{div}(\theta \mathbf{B})+\mathbf{b} \\
\left.\mathbf{u}\right|_{\partial \Omega}=0
\end{array}\right.
$$

We take the $L^{2}$-norm on both sides of the former equation. Thus

$$
\begin{aligned}
\|\mathbb{A} \mathbf{u}\| & \leq\left\|\mathcal{P}\left[\mathbf{u}_{t}+[\mathbf{u} \cdot \nabla] \mathbf{u}\right]\right\|+\alpha_{1}\|\mathcal{P} \operatorname{div}(\theta \mathbf{B})\|+\|\mathbf{b}\| \\
& \leq\left\|\mathbf{u}_{t}\right\|+C\|\nabla \mathbf{u}\|^{3 / 2}\left\|\partial^{2} \mathbf{u}\right\|^{1 / 2}+C\|\nabla \theta\|^{1 / 2}\|\theta\|_{2}^{1 / 2}\|\mathbf{B}\|_{2}+\|\mathbf{b}\|,
\end{aligned}
$$

which combined with (4.4) and (4.20) leads to

$$
\|\mathbb{A} \mathbf{u}\| \leq\left\|\mathbf{u}_{t}\right\|+C\|\nabla \mathbf{u}\|^{3}+C\|\nabla \theta\|^{1 / 2}\|\theta\|_{2}^{1 / 2}\|\mathbf{B}\|_{2}+\|\mathbf{b}\| .
$$

We now turn to the estimate of $\|\theta\|_{2}$. We first notice that $\theta$ satisfies

$$
\left\{\begin{array}{l}
-\kappa \triangle \theta=-\theta_{t}-[\mathbf{u} \cdot \nabla] \theta+\alpha_{1} \theta \mathbf{B}: \mathbf{D}+2 \nu \mathbf{D}: \mathbf{D}+r \quad \text { in } \Omega \\
\left.\theta\right|_{\partial \Omega}=0
\end{array}\right.
$$


Thus from the regularity result (Ref. 3, p. 181), one has

$$
\begin{aligned}
\|\theta\|_{2} \leq & C\left\|\theta_{t}\right\|+C\|[\mathbf{u} \cdot \nabla] \theta\|+C\|\theta \mathbf{D}: \mathbf{B}\|+2 \nu\|\mathbf{D}: \mathbf{D}\|+C\|r\| \\
\leq & C\left\|\theta_{t}\right\|+C\|\nabla \mathbf{u}\|\|\nabla \theta\|^{1 / 2}\|\theta\|_{2}^{1 / 2}+C\|\nabla \theta\|^{1 / 2}\|\theta\|_{2}^{1 / 2}\|\nabla \mathbf{u}\|\|\mathbf{B}\|_{2} \\
& +C\|\nabla \mathbf{u}\|_{1}^{3 / 2}\|\nabla \mathbf{u}\|^{1 / 2}+C\|r\| .
\end{aligned}
$$

By (4.20) and (4.4), we then find that

$$
\begin{aligned}
\|\theta\|_{2} \leq & C\left\|\theta_{t}\right\|+C\|\nabla \mathbf{u}\|^{2}\|\nabla \theta\|+C\|\nabla \theta\|\|\nabla \mathbf{u}\|^{2}\|\mathbf{B}\|_{2}^{2} \\
& +C\|\mathbb{A} \mathbf{u}\|^{3 / 2}\|\nabla \mathbf{u}\|^{1 / 2}+C\|r\| .
\end{aligned}
$$

Returning to (6.11) with (6.12), and (4.20), we obtain

$$
\begin{aligned}
\|\mathbb{A} \mathbf{u}\| \leq & \left\|\mathbf{u}_{t}\right\|+C\|\nabla \mathbf{u}\|^{3}+C\|\nabla \theta\|^{2}\|\mathbf{B}\|_{2}^{4}\|\nabla \mathbf{u}\|+\|\mathbf{b}\| \\
& +C\left\|\theta_{t}\right\|^{1 / 2}\|\nabla \theta\|^{1 / 2}\|\mathbf{B}\|_{2}+C\|\nabla \mathbf{u}\|\|\nabla \theta\|\|\mathbf{B}\|_{2} \\
& +C\|\nabla \theta\|\|\nabla \mathbf{u}\|\|\mathbf{B}\|_{2}^{2}+\|r\|^{1 / 2}\|\nabla \theta\|^{1 / 2}\|\mathbf{B}\|_{2}
\end{aligned}
$$

Therefore (6.12) and (6.10) become respectively

$$
\begin{aligned}
\|\theta\|_{2} \leq & C\left\|\theta_{t}\right\|+C\|\nabla \mathbf{u}\|^{2}\|\nabla \theta\|+C\|\nabla \theta\|\|\nabla \mathbf{u}\|^{2}\|\mathbf{B}\|_{2}^{2}+C\|\nabla \mathbf{u}\|^{5} \\
& +C\|\nabla \mathbf{u}\|^{1 / 2}\left\|\mathbf{u}_{t}\right\|^{3 / 2}+C\|\nabla \theta\|^{3}\|\mathbf{B}\|_{2}^{6}\|\nabla \mathbf{u}\|^{2}+C\|\nabla \mathbf{u}\|^{1 / 2}\|\mathbf{b}\|^{3 / 2} \\
& +C\|\nabla \mathbf{u}\|^{1 / 2}\left\|\theta_{t}\right\|^{3 / 4}\|\nabla \theta\|^{3 / 4}\|\mathbf{B}\|_{2}^{3 / 4}+\|\nabla \mathbf{u}\|^{2}\|\nabla \theta\|^{3 / 2}\|\mathbf{B}\|_{2}^{3 / 2} \\
& +C\|\nabla \theta\|^{3 / 2}\|\nabla \mathbf{u}\|^{2}\|\mathbf{B}\|_{2}^{3}+C\|r\|^{3 / 4}\|\nabla \mathbf{u}\|^{1 / 2}\|\nabla \theta\|^{3 / 4}\|\mathbf{B}\|_{2}^{3 / 2}+C\|r\|
\end{aligned}
$$

and

$$
\begin{aligned}
\|\mathbf{u}\|_{3} \leq & C\|\mathbf{b}\|_{1}+C\left\|\nabla \mathbf{u}_{t}\right\|+C\|\nabla \mathbf{u}\|^{2}+C\|\nabla \mathbf{u}\|^{4} \\
& +C\left\{\left\|\theta_{t}\right\|+\|\nabla \mathbf{u}\|^{2}\|\nabla \theta\|+C\|\nabla \theta\|\|\nabla \mathbf{u}\|^{2}\|\mathbf{B}\|_{2}^{2}+C\|\nabla \mathbf{u}\|^{5}\right\}\|\mathbf{B}\|_{2} \\
& +C\left\{\|\nabla \mathbf{u}\|^{1 / 2}\left\|\mathbf{u}_{t}\right\|^{3 / 2}+c\|\nabla \theta\|^{3}\|\mathbf{B}\|_{2}^{6}\|\nabla \mathbf{u}\|^{2}+C\|\nabla \mathbf{u}\|^{1 / 2}\|\mathbf{b}\|^{3 / 2}\right\}\|\mathbf{B}\|_{2} \\
& +C\left\{\|\nabla \mathbf{u}\|^{1 / 2}\left\|\theta_{t}\right\|^{3 / 4}\|\nabla \theta\|^{3 / 4}\|\mathbf{B}\|_{2}^{3 / 4}+\|\nabla \mathbf{u}\|^{2}\|\nabla \theta\|^{3 / 2}\|\mathbf{B}\|_{2}^{3 / 2}\right\}\|\mathbf{B}\|_{2} \\
& +C\left\{\|\nabla \theta\|^{3 / 2}\|\nabla \mathbf{u}\|^{2}\|\mathbf{B}\|_{2}^{3}+\|r\|^{3 / 4}\|\nabla \mathbf{u}\|^{1 / 2}\|\nabla \theta\|^{3 / 4}\|\mathbf{B}\|_{2}^{3 / 2}+\|r\|\right\}\|\mathbf{B}\|_{2} \\
& +C\left\{\left\|\mathbf{u}_{t}\right\|^{2}+\|\nabla \mathbf{u}\|^{6}+\|\nabla \theta\|^{4}\|\mathbf{B}\|_{2}^{8}\|\nabla \mathbf{u}\|^{2}\right\} \\
& +C\left\{\left\|\theta_{t}\right\|\|\nabla \theta\|\|\mathbf{B}\|_{2}^{2}+\|\nabla \mathbf{u}\|^{2}\|\nabla \theta\|^{2}\|\mathbf{B}\|_{2}^{2}\right\} \\
& +C\left\{\|\nabla \theta\|^{2}\|\nabla \mathbf{u}\|^{2}\|\mathbf{B}\|_{2}^{4}+\|\nabla \theta\|^{2}\|\mathbf{B}\|_{2}^{4}\right\}+C\|\mathbf{b}\|^{2}+C\|r\|^{2} .
\end{aligned}
$$


Returning to (6.9) with (6.14) and (6.15), we obtain

$$
\begin{aligned}
X_{t}+ & C\left\|\nabla \mathbf{u}_{t}\right\|^{2}+C\|\mathbb{A} \mathbf{u}\|^{2}+C\left\|\nabla \theta_{t}\right\|^{2}+C\|\Delta \theta\|^{2} \\
& \leq C\left\{X^{2}+X^{3}+X^{4}+\cdots+X^{32}\right\}+F
\end{aligned}
$$

where

$$
\begin{aligned}
X(t)= & 1+\|\mathbf{B}(t)\|_{2}^{2}+\left\|\mathbf{B}_{t}(t)\right\|^{2}+\|\nabla \mathbf{u}(t)\|^{2}+\left\|\mathbf{u}_{t}(t)\right\|^{2} \\
& +\|\nabla \theta(t)\|^{2}+\left\|\theta_{t}(t)\right\|^{2}, \\
F= & F\left(\left\|r_{t}\right\|,\|r\|,\left\|\mathbf{b}_{t}\right\|,\|\mathbf{b}\|_{1}\right) .
\end{aligned}
$$

Since $\theta \in H^{2}(\Omega) \cap H_{0}^{1}(\Omega)$, then there is a positive constant $C$, such that $C\|\nabla \theta\| \leq$ $\|\triangle \theta\|$. But $\|\nabla \mathbf{u}\| \leq\|\mathbb{A} \mathbf{u}\|$ since $\|\mathbb{A} \mathbf{u}\|$ and $\|\mathbf{u}\|_{2}$ are equivalent (see (4.4)). Next, since $\left.\theta_{t}\right|_{\partial \Omega}=0$, Poincaré's inequality ensures that $\left\|\theta_{t}(t)\right\| \leq C\left\|\nabla \theta_{t}(t)\right\|$. Now, adding and subtracting $\|\mathbf{B}(t)\|_{2}^{2}+\left\|\mathbf{B}_{t}(t)\right\|^{2}$ from (6.16), and taking into account the facts just mentioned, one obtains

$$
X_{t}+C_{1} X \leq C_{2}\left\{X^{2}+X^{3}+X^{4}+\cdots+X^{32}\right\}+\underbrace{C+F}_{G} .
$$

\section{Step 2. Application of the differential inequality (6.18)}

First, one observes that for the local existence (see Theorem 5.1), the lifetime of the solution $T_{0}$ depends $\left\|\mathbb{A}_{0}\right\|,\left\|\mathbf{B}_{0}\right\|_{2}$ and $\left\|\theta_{0}\right\|_{1}$, when $\mathbf{b}$ and $r$ satisfies the conditions listed in Theorem 5.1.

We now recall one of the fundamental tools in getting global existence.

Lemma 6.1. (Ref. 9) Let $f$ be a non-negative, absolutely continuous function satisfying the inequality

$$
f^{\prime}+k f \leq \alpha\left(f^{2}+f^{3}+\cdots+f^{2 m}\right)+\beta
$$

where $m \geq 2, k>0, \alpha>0$ and $\beta \geq 0$ are some constants. Let $M, 0<M<M_{0}$ is the unique positive solution of

$$
M^{2 m-1}+\cdots+M^{3}+M^{2}+M-\frac{k}{2 \alpha}=0 .
$$

If $f(0) \leq M$ and $\beta \leq \frac{k}{2} M$, then $f(t)$ is bounded by $M$ for all $t>0$.

From (6.2)-(6.5) and (6.8), one readily checks that the real-valued function $t \mapsto \mathbf{X}$ given by $(6.18)_{1}$ is absolutely continuous on $\left[0, T_{0}\right]$.

Next, since $X(t)$ is the solution of the differential inequality (6.18), one has

$$
X(0)=\left\|\mathbf{B}_{0}\right\|_{2}^{2}+\left\|\mathbf{B}_{t}(0)\right\|^{2}+\left\|\nabla \mathbf{u}_{0}\right\|^{2}+\left\|\mathbf{u}_{t}(0)\right\|^{2}+\left\|\nabla \theta_{0}\right\|^{2}+\left\|\theta_{t}(0)\right\|^{2} .
$$

But from (3.3), we can deduce that

$$
\begin{aligned}
\left\|\mathbf{u}_{t}(0)\right\| & \leq\left\|\left[\mathbf{u}_{0} \cdot \nabla\right] \mathbf{u}_{0}\right\|+\nu\left\|\triangle \mathbf{u}_{0}\right\|+\alpha_{1}\left\|\operatorname{div}\left(\theta_{0} \mathbf{B}_{0}\right)\right\|+\|\mathbf{b}(0)\| \\
& \leq C\left\|\mathbf{u}_{0}\right\|_{2}^{2}+C\left\|\mathbf{u}_{0}\right\|_{2}+C\left\|\theta_{0}\right\|_{2}\left\|\mathbf{B}_{0}\right\|_{2}+\|\mathbf{b}(0)\|,
\end{aligned}
$$




$$
\begin{aligned}
\left\|\mathbf{B}_{t}(0)\right\| & \leq\left\|\left[\mathbf{u}_{0} \cdot \nabla\right] \mathbf{B}_{0}\right\|+\left\|\left[\nabla \mathbf{u}_{0}\right] \mathbf{B}_{0}\right\|+C\left\|\frac{\theta_{0}}{\operatorname{tr} \mathbf{B}_{0}^{-1}}\right\|+\left\|\theta_{0} \mathbf{B}_{0}\right\| \\
& \leq C\left\|\mathbf{u}_{0}\right\|_{1}\left\|\mathbf{B}_{0}\right\|_{2}+\left\|\mathbf{u}_{0}\right\|_{2}\left\|\mathbf{B}_{0}\right\|_{2}+C\left\|\theta_{0}\right\|+C\left\|\theta_{0}\right\|_{1}\left\|\mathbf{B}_{0}\right\|_{1}, \\
\left\|\theta_{t}(0)\right\| & \leq\left\|\left(\mathbf{u}_{0} \cdot \nabla\right) \theta_{0}\right\|+\kappa\left\|\triangle \theta_{0}\right\|+C\left\|\theta_{0} \mathbf{B}_{0}: \nabla \mathbf{u}_{0}\right\|+C\left\|\nabla \mathbf{u}_{0}: \nabla \mathbf{u}_{0}\right\|+\|r(0)\| \\
& \leq C\left\|\mathbf{u}_{0}\right\|_{1}\left\|\theta_{0}\right\|_{2}+C\left\|\theta_{0}\right\|_{2}+C\left\|\theta_{0}\right\|_{2}\left\|\mathbf{B}_{0}\right\|_{2}\left\|\nabla \mathbf{u}_{0}\right\|+C\left\|\mathbf{u}_{0}\right\|_{2}^{2}+\|r(0)\|,
\end{aligned}
$$

which shows that $X(0)$ is bounded.

From (6.18), (6.18) and Lemma 6.1, there exists a positive constant $M_{0}$, such that if $X(0) \leq M<M_{0}$, and $G \leq C_{1} M / 2$, then $X(t) \leq M$ for all $t>0$ as soon as $\mathbf{u}(t), \theta(t)$ and $\mathbf{B}(t)$ satisfy the regularity listed in Theorem 5.1. From (6.13)(6.15) and the definition of $X$, we then deduce that $\|\mathbb{A} \mathbf{u}(t)\|,\|\mathbf{B}(t)\|_{2},\|\theta(t)\|_{2}$ are uniformly bounded on the maximal interval of existence of $(\mathbf{u}, p, \mathbf{B}, \theta)$. Thus the local solution constructed in Theorem 5.1 can be extended for all positive time. This completes the proof of Theorem 6.1.

\section{Acknowledgment}

We acknowledge helpful comments and suggestions from Prof. Niko Sauer. Our thanks are also addressed to the referees for pertinent remarks that have led to some improvements of this study. B.D.R. acknowledges the Department of Science and Technology and the National Research Foundation, through the South African Research Chairs Initiative.

\section{References}

1. M. Anand and K. R. Rajagopal, A shear-thinning viscoelastic fluid model for describing the flow of blood, Int. J. Cardiovascular Med. Sci. 4 (2004) 59-68.

2. H. A. Barnes, J. Hutton and K. Walters, An Introduction to Rheology (Elsevier, 1989).

3. H. Brézis, Analyse Fonctionelle Linéaire: Théorie et Applications (Mason, 1983).

4. P. Chadwick, Continuum Mechanics: Concise Theory and Problems (Dover, 1999) (corrected and enlarged version of 1976 edition).

5. J. Y. Chemin and N. Masmoudi, About lifespan of regular solutions of equations related to viscoelastic fluids, SIAM J. Math. Anal. 33 (2001) 84-112.

6. L. C. Evans, Partial Differential Equations (Amer. Math. Soc., 1998).

7. G. P. Galdi and B. D. Reddy, Well-posedness of the problem of fibre suspension flows, J. Non-Newtonian Fluid Mech. 83 (1999) 205-230.

8. V. Girault and P. A. Raviart, Finite Element Methods for Navier-Stokes Equations. Theory and Algorithms, Springer Series in Computational Mathematics, Vol. 5 (Springer-Verlag, 1986).

9. C. Guillopé and J. C. Saut, Existence results for the flow of viscoelastic fluids with a differential constitutive law, Nonlinear Anal. Theory, Methods Appl. 15 (1990) 849-869.

10. W. Han and B. D. Reddy, Plasticity: Mathematical Theory and Numerical Analysis (Springer, 1999).

11. A. Jeffrey, Quasilinear Hyperbolic Systems and Waves (Pitman, 1976). 
12. K. Kannan, I. J. Rao and K. R. Rajagopal, A thermomechanical framework for the glass transition phenomenon in certain polymers and its application to fiber spinning, J. Rheology 46 (2002) 977-999.

13. K. Kannan and K. R. Rajagopal, Simulation of fiber spinning including flow-induced crystallization, J. Rheology 49 (2005) 683-703.

14. S. Karra and K. R. Rajagopal, A thermomechanical framework to develop rate-type models for fluids without instantaneous elasticity, Acta Mech. 205 (2009) 105-119.

15. F.-H. Lin and C. Liu, Nonparabolic dissipative systems modeling the flow of liquid crystals, Comm. Pure Appl. Math. 48 (1995) 501-537.

16. F.-H. Lin and P. Zhang, On the initial boundary value problem of the incompressible viscoelastic fluid system, Comm. Pure Appl. Math. LXI (2008) 539-558.

17. F.-H. Lin, C. Liu and P. Zhang, On the hydrodynamics of viscoelastic fluids, Comm. Pure Appl. Math. LVIII (2005) 1437-1471.

18. J. L. Lions, Quelques Méthodes de Résolution des Problèmes aux Limites Non Linéaires (Masson, 1969).

19. P. L. Lions and N. Masmoudi, Global solutions for some Oldroyd models of nonNewtonian flows, Chin. Ann. Math., Ser. B 21 (2000) 131-146.

20. J. Malek, J. Nečas and K. R. Rajagopal, Global analysis of the flow of fluids with pressure dependent viscosities, Arch. Rational Mech. Anal. 165 (2002) 243-269.

21. J. Malek, J. Nečas and K. R. Rajagopal, Global existence of solutions for flows of fluids with pressure and shear dependent viscosities, Appl. Math. Lett. 15 (2002) 961-967.

22. L. Molinet and R. Talhouk, Existence and stability results for 3D regular flows of viscoelastic fluids of White-Metzner type, Nonlinear Anal. 58 (2004) 813-833.

23. J. M. W. Munganga and B. D. Reddy, Local and global existence of solutions to equations for flows of fibre suspensions, Math. Models Methods Appl. Sci. 12 (2002) $1177-1203$.

24. R. G. Owens and T. N. Philips, Computational Rheology (Imperial College Press, 2002).

25. K. R. Rajagopal and A. R. Srinivasa, Mechanics of the inelastic behavior of materials. Parts I: Theoretical underpinnings, Int. J. Plasticity 118 (1998) 945-967.

26. K. R. Rajagopal and A. R. Srinivasa, A Gibbs potential based formulation for obtaining the response functions for a class of viscoelastic materials, Proc. Royal Soc. Ser. A 467 (2011) 39-58.

27. I. J. Rao and K. R. Rajagopal, A thermomechanical framework for the study of crystallization in polymers, Z Angew. Math. Phys. (ZAMP) 53 (2002) 365-406.

28. T. C. Sideris and B. Thomases, Global existence for three-dimensional incompressible isotropic elastodynamics via the incompressible limit, Comm. Pure Appl. Math. 58 (2005) 750-788.

29. J. Simon, Compact set in the space $L^{p}(0, T ; B)$, Ann. Mat. Pura Appl. 146 (1987) 65-96.

30. R. Temam, Navier-Stokes Equations: Theory and Numerical Analysis (Amer. Math. Soc., 2001) (reprint of the 1984 edition).

31. R. Temam, Infinite Dynamical Systems in Mechanics and Physics, 2nd edn. (SpringerVerlag, 1998). 